\title{
Approximating Edit Distance Within Constant Factor in Truly Sub-Quadratic Time
}

\author{
Diptarka Chakraborty*1, Debarati Das ${ }^{\dagger 2}$, Elazar Goldenberg ${ }^{\ddagger 3}$, Michal Koucký ${ }^{\$ 4}$, and \\ Michael Saks 15 \\ ${ }^{1}$ School of Computing, National University of Singapore, Singapore \\ ${ }^{2}$ University of Copenhagen, Denmark \\ ${ }^{3}$ School of Computer Science, The Academic College of Tel Aviv-Yaffo, Israel \\ ${ }^{4}$ Computer Science Institute of Charles University, Prague, Czech Republic \\ ${ }^{5}$ Department of Mathematics, Rutgers University, Piscataway, NJ, USA
}

\begin{abstract}
Edit distance is a measure of similarity of two strings based on the minimum number of character insertions, deletions, and substitutions required to transform one string into the other. The edit distance can be computed exactly using a dynamic programming algorithm that runs in quadratic time. Andoni, Krauthgamer and Onak (2010) gave a nearly linear time algorithm that approximates edit distance within approximation factor poly $(\log n)$.

In this paper, we provide an algorithm with running time $\widetilde{O}\left(n^{2-2 / 7}\right)$ that approximates the edit distance within a constant factor.
\end{abstract}

*diptarka@comp.nus.edu.sg

†debaratix710@gmail.com

${ }^{\ddagger}$ elazargo@mta.ac.il

${ }^{\S}$ koucky@iuuk.mff.cuni.cz

Imsaks30@gmail.com 


\section{Introduction}

Exact computation of edit distance. The edit distance (aka Levenshtein distance) [22] between strings $x, y$, denoted by $d_{\text {edit }}(x, y)$, is the minimum number of character insertions, deletions, and substitutions needed to convert $x$ into $y$. It is a widely used distance measure between strings that finds applications in fields such as computational biology, pattern recognition, text processing, and information retrieval. The problems of efficiently computing $d_{\text {edit }}(x, y)$, and of constructing an optimal alignment (sequence of operations that converts $x$ to $y$ ), are of significant interest.

Edit distance can be evaluated exactly in quadratic time via dynamic programming (Wagner and Fischer [27]). Landau et al. [21] gave an algorithm that finds an optimal alignment in time $O\left(n+d_{\text {edit }}(x, y)^{2}\right)$, improving on a previous $O\left(n \cdot d_{\text {edit }}(x, y)\right)$ algorithm of Ukkonen [26]. Masek and Paterson 23] obtained the first (slightly) sub-quadratic $O\left(n^{2} / \log n\right)$ time algorithm, and the current asymptotically fastest algorithm (Grabowski [19]) runs in time $O\left(n^{2} \log \log n / \log ^{2} n\right)$. Backurs and Indyk [9] showed that a truly sub-quadratic algorithm $\left(O\left(n^{2-\delta}\right)\right.$ for some $\left.\delta>0\right)$ would imply a $2^{(1-\gamma) n}$ time algorithm for CNF-satisfiability, contradicting the Strong Exponential Time Hypothesis (SETH). Abboud et al. [3] showed that even shaving an arbitrarily large polylog factor from $n^{2}$ would have the plausible, but apparently hard-to-prove, consequence that NEXP does not have non-uniform $N C^{1}$ circuits. For further "barrier" results, see [2, 16].

Approximation algorithms. There is a long line of work on approximating edit distance. The exact $O\left(n+d_{\text {edit }}(x, y)^{2}\right)$ time algorithm of Landau et al. [18, 21, 25] yields a linear time $\sqrt{n}$-factor approximation. This approximation factor was improved, first to $n^{3 / 7}\left[10\right.$, then to $n^{1 / 3+o(1)}[12]$ and later to $2^{\widetilde{O}(\sqrt{\log n})}$ [8], all with slightly superlinear running time. Batu et al. [11] provided an $O\left(n^{1-\alpha}\right)$-approximation algorithm with running time $O\left(n^{\max \left\{\frac{\alpha}{2}, 2 \alpha-1\right\}}\right)$. The strongest result of this type is the $(\log n)^{O(1 / \epsilon)}$-factor approximation (for every $\epsilon>0$ ) with running time $n^{1+\epsilon}$ of Andoni et al. [5]. Abboud and Backurs [1] showed that a truly sub-quadratic deterministic time 1+o(1)-factor approximation algorithm for edit distance would imply new circuit lower bounds.

Independent of our work, Boroujeni et al. [13] obtained a truly sub-quadratic quantum algorithm that provides a constant factor approximation. Their latest results [14] are a $(3+\epsilon)$-factor with running time $\widetilde{O}\left(n^{2-4 / 21} / \epsilon^{O(1)}\right)$ and a faster $\widetilde{O}\left(n^{1.708}\right)$-time with a larger constant factor approximation.

Andoni and Nguyen [6] found a randomized algorithm that approximates Ulam distance of two permutations of $\{1, \ldots, n\}$ (edit distance with only insertions and deletions) within a (large) constant factor in time $\widetilde{O}\left(\sqrt{n}+n / d_{\text {Ulam }}(x, y)\right)$, where $d_{\text {Ulam }}(x, y)$ is the Ulam distance of the input; this was improved by Naumovitz et al. 24] to a $(1+\varepsilon)$-factor approximation (for any $\varepsilon>0$ ) with similar running time.

Our results. We present the first truly sub-quadratic time classical algorithm that approximates edit distance within a constant factor.

Theorem 1.1. There is a randomized algorithm ED-UB that on input strings $x, y$ of length $n$ over any alphabet $\Sigma$ outputs an upper bound on $d_{\text {edit }}(x, y)$ in time $\widetilde{O}\left(n^{12 / 7}\right)$ that, with probability at least $1-n^{-5}$, is at most a fixed constant multiple of $d_{\text {edit }}(x, y)$.

If the output is $U$, then the algorithm has implicitly found an alignment of cost at most $U$. The algorithm can be modified to explicitly output such an alignment.

The approximation factor proved in this preliminary version is 1680 , can be greatly improved by tweaking parameters. We believe, but have not proved, that with sufficient care the algorithm can be modified (with no significant increase in the running time) to get $(3+\epsilon)$-approximation. 
Theorem 1.1 follows from:

Theorem 1.2. For every $\theta \in\left[n^{-1 / 5}, 1\right]$, there is a randomized algorithm $\mathbf{G A P - U B} \mathbf{B}_{\theta}$ that on input strings $x, y$ of length $n$ outputs $u=\mathbf{G A P}-\mathbf{U B}_{\theta}(x, y)$ such that: (1) $d_{\text {edit }}(x, y) \leq u$ and (2) on any input with $d_{\text {edit }}(x, y) \leq \theta n, u \leq 840 \theta n$ with probability at least $1-n^{-7}$. The running time of GAP-UB $_{\theta}$ is $\tilde{O}\left(n^{2-2 / 7} \theta^{4 / 7}\right)$.

The name GAP-UB $\mathbf{U B}_{\theta}$ reflects that this is a "gap algorithm", which distinguishes inputs with $d_{\text {edit }}(x, y) \leq \theta n$ (where the output is at most $840 \theta n$ ), and those with $d_{\text {edit }}(x, y)>840 \theta n$ (where the output is greater than $840 \theta n)$.

Theorem 1.1 follows via a routine construction of ED-UB from GAP-UB ${ }_{\theta}$, presented in Section 5. The rest of the paper is devoted to proving Theorem 1.2 .

Further developments. Since the initial circulation of our paper, improved algorithms for approximating edit distance have been obtained building on our techniques. For every $\epsilon>0$, Andoni [4] presents an algorithms that computes a constant factor approximation to edit distance in time $O\left(n^{\frac{3}{2}+\epsilon}\right)$. Brakensiek and Rubinstein [15] and independently Koucký and Saks [20] give for each $\epsilon>0$, an algorithm running in time $O\left(n^{1+\epsilon}\right)$ that computes a constant factor approximation to edit distance with additive error $n^{1-\beta}$, for some $\beta>0$. Eventually, Andoni and Nosatzki [7] give for each $\epsilon>0$, an algorithm running in time $O\left(n^{1+\epsilon}\right)$ that computes a constant factor approximation to edit distance of any two strings.

The framework of the algorithm. We use a standard two-dimensional representation of edit distance. Visualize $x$ as lying on a horizontal axis and $y$ as lying on a vertical axis, with horizontal coordinate $i \in\{1, \ldots, n\}$ corresponding to $x_{i}$ and vertical component $j$ corresponding to $y_{j}$. The width $\mu(I)$ of interval $I \subseteq\{0,1, \ldots, n\}$ is $\max (I)-\min (I)=|I|-1$. Also, $x_{I}$ denotes the substring of $x$ indexed by $I \backslash\{\min (I)\}$. (Note: $x_{\min (I)}$ is not part of $x_{I}$, e.g., $x_{\{0, \ldots, n\}}=x$. This convention is motivated by Proposition 1.3.) We refer to $I$ as an $x$-interval to indicate that it indexes a substring of $x$, and $J$ as a $y$-interval to indicate that it indexes a substring of $y$. A box is a set $I \times J$ where $I$ is an $x$-interval and $J$ is a $y$-interval; $I \times J$ corresponds to the substring pair $\left(x_{I}, y_{J}\right) . I \times J$ is a $w$-box if $\mu(I)=\mu(J)=w$. We often abbreviate $d_{\text {edit }}\left(x_{I}, y_{J}\right)$ by $d_{\text {edit }}(I, J)$. A decomposition of an $x$-interval $I$ is a sequence $I_{1}, \ldots, I_{\ell}$ of subintervals with $\min \left(I_{1}\right)=\min (I), \max \left(I_{\ell}\right)=\max (I)$ and for $j \in[\ell-1], \max \left(I_{j}\right)=\min \left(I_{j+1}\right)$. If all the subintervals $I_{1}, \ldots, I_{\ell}$ are of the same width $w$ then it is the $w$-decomposition of $I$ denoted by $\mathcal{I}_{w}(I) .\left(\mathcal{I}_{w}(\{0, \ldots, n\})\right.$ is denoted by $\mathcal{I}_{w}$.)

Associated to $x, y$ is a directed graph $G_{x, y}$ with edge costs called a grid graph with vertex set $\{0, \ldots, n\} \times\{0, \ldots, n\}$ and all edges of the form $(i-1, j) \rightarrow(i, j)(H$-steps $),(i, j-1) \rightarrow(i, j)$ ( $V$-steps) and $(i-1, j-1) \rightarrow(i, j)$ ( $D$-steps). Every H-step or V-step costs 1 , and D-steps cost 1 if $x_{i} \neq y_{j}$ and 0 otherwise. There is a 1-1 correspondence that maps a path from $(0,0)$ to $(n, n)$ (source-sink path) to an alignment from $x$ to $y$, i.e. a sequence of character deletions, insertions and substitutions that changes $x$ to $y$, where an H-step $(i-1, j) \rightarrow(i, j)$ means "delete $x_{i}$ ", a V-step $(i, j-1) \rightarrow(i, j)$ means "insert $y_{j}$ between $x_{i}$ and $x_{i+1}$ " and a D-step $(i-1, j-1) \rightarrow(i, j)$ means replace $x_{i}$ by $y_{j}$, unless they are already equal. We have:

Proposition 1.3. The cost of an alignment is the sum of edge costs of its associated path $\tau$, $\operatorname{cost}(\tau)$, and $d_{\text {edit }}(x, y)$ is equal to $\operatorname{cost}\left(G_{x, y}\right)$, the minimal cost of a path from $(0,0)$ to $(n, n)$.

For $I, J \subseteq\{0, \ldots, n\}, G_{x, y}(I \times J) \cong G_{x_{I}, y_{J}}$ is the grid graph induced on $I \times J$, and $d_{\text {edit }}(I, J)=$ $\operatorname{cost}\left(G_{x, y}(I \times J)\right)$. The natural high-level idea of $\mathbf{G A P}-\mathbf{U B}_{\theta}$ appears (explicitly or implicitly) in previous work. The algorithm has two phases. First, the covering phase identifies a set $\mathcal{R}$ of 
certified boxes which are pairs $(I \times J, \kappa)$, where $\kappa$ is an upper bound on the normalized edit distance $\Delta_{\text {edit }}\left(x_{I}, y_{J}\right)=d_{\text {edit }}\left(x_{I}, y_{J}\right) / \mu(I)$. $\left(\Delta_{\text {edit }}(I, J)\right.$ is more convenient than $d_{\text {edit }}(I, J)$ for the covering phase.) Second, the min-cost path phase, takes input $\mathcal{R}$ and uses a straightforward customized variant of dynamic programming to find an upper bound $U(\mathcal{R})$ on $d_{\text {edit }}(x, y)$ in time quasilinear in $|\mathcal{R}|$. The central issue is to ensure that the covering phase outputs $\mathcal{R}$ that is sufficiently informative so that $U(\mathcal{R}) \leq c \cdot d_{\text {edit }}(x, y)$ for constant $c$, while running in sub-quadratic time.

Simplifying assumptions. The input strings $x, y$ have equal length $n$. (It is easy to reduce to this case: pad the shorter string to the length of the longer using a new symbol. The edit distance of the new pair is between the original edit distance and twice the original edit distance. This factor 2 increase in approximation factor can be avoided by generalizing our algorithm to the case $|x| \neq|y|$, but we won't do this here.) We assume $n$ is a power of 2 (by padding both strings with a new symbol, which leaves edit distance unchanged). We assume that $\theta$ is a (negative) integral power of 2. The algorithm involves integer parameters $w_{1}, w_{2}, d$, all of which are chosen to be powers of 2 .

Organization of the paper. Section 2 is a detailed overview of the covering phase algorithm and its analysis. Section 3 presents the pseudo-code and analysis for the covering phase. Section 4 presents the min-cost path phase algorithm. Section 5 summarizes the full algorithm and discusses improvements in running time via recursion.

\section{Covering algorithm: Detailed overview}

We give a detailed overview of the covering phase and its time analysis and proof of correctness, ignoring minor technical details. The pseudo-code in Section 3 corresponds to the overview, with technical differences mainly to improve running time. We will illustrate the sub-quadratic time analysis with threshold parameter $\theta=n^{-1 / 50}$ and algorithm parameters $w_{1}=n^{1 / 10}, w_{2}=n^{3 / 10}$ and $d=n^{1 / 5}$.

The covering phase outputs a set $\mathcal{R}$ of certified boxes. The goal is that $\mathcal{R}$ includes an adequate approximating sequence for some min-cost path $\tau$ in $G_{x, y}$, which is a sequence $\sigma$ of certified boxes $\left(I_{1} \times J_{1}, \kappa_{1}\right), \ldots,\left(I_{\ell} \times J_{\ell}, \kappa_{\ell}\right)$ that satisfies:

1. $I_{1}, \ldots, I_{\ell}$ is a decomposition of $\{0, \ldots, n\}$.

2. $I_{i} \times J_{i}$ is an adequate cover of $\tau_{i}$, where $\tau_{i}=\tau_{I_{i}}$ denotes the minimal subpath of $\tau$ whose projection to the $x$-axis is $I_{i}$, and adequate cover means that the (vertical) distance from the start vertex (resp. final vertex) of $\tau_{i}$ and the lower left (resp. upper right) corner of $G_{x, y}\left(I_{i} \times J_{i}\right)$, is at most a constant multiple of $\operatorname{cost}\left(\tau_{i}\right)+\theta \mu\left(I_{i}\right)$.

3. The sequence $\sigma$ is adequately bounded, i.e., $\sum_{i} \mu\left(I_{i}\right) \kappa_{i} \leq c(\operatorname{cost}(\tau)+\theta n)$, for a constant $c$.

This is a slight oversimplification of Definition 3. (See Figure 1 for an illustration.)

The intuition for the second condition is that $\tau_{i}$ is "almost" a path between the lower left and upper right corners of $I_{i} \times J_{i}$. Now $\tau_{i}$ might have a vertical extent $J^{\prime}$ that is much larger than its horizontal extent $I_{i}$, in which case it is impossible to place a square $I_{i} \times J_{i}$ with corners close to both endpoints of $\tau_{i}$. But in that case, $\tau_{i}$ has a very high cost (at least $\left.\left|\mu\left(J^{\prime}\right)-\mu\left(I_{i}\right)\right|\right)$. The closeness required is adjusted based on $\operatorname{cost}\left(\tau_{i}\right)$, with relaxed requirements if $\operatorname{cost}\left(\tau_{i}\right)$ is large.

The output of the min-cost path phase should satisfy the requirements of GAP-UB ${ }_{\theta}$. Roughly speaking, Lemma 4.1 shows that if the min-cost path phase receives $\mathcal{R}$ that contains an adequate approximating sequence to some min-cost path $\tau$, then it will output an upper bound to $d_{\text {edit }}(x, y)$ 
that is at most $c\left(d_{\text {edit }}(x, y)+\theta n\right)$ for some $c$. So that on input $x, y$ with $d_{\text {edit }}(x, y) \leq \theta n$, the output is at most $2 c \theta n$, satisfying the requirements of $\mathbf{G A P}-\mathbf{U B}_{\theta}$. This formalizes the intuition that an adequate approximating sequence captures enough information to deduce a good bound on $\operatorname{cost}(\tau)$.

Once and for all, we fix a min-cost path $\tau$. Our task for the covering phase is that, with high probability, $\mathcal{R}$ includes an adequate approximating sequence for $\tau$.

A $\tau$-match for an $x$-interval $I$ is a $y$-interval $J$ such that $I \times J$ is an adequate cover of $\tau_{I}$. It is easy to show (Proposition 3.3) that this implies $d_{\text {edit }}(I, J) \leq O\left(\operatorname{cost}\left(\tau_{I}\right)+\theta \mu(I)\right)$. A box $I \times J$ is said to be $\tau$-compatible if $J$ is a $\tau$-match for $I$ and a box sequence is $\tau$-compatible if every box is $\tau$-compatible. A $\tau$-compatible certified box sequence whose distance upper bounds are (on average) within a constant factor of the actual cost, satisfies the requirements for an adequate approximating sequence. Our cover algorithm will ensure that $\mathcal{R}$ contains such a sequence.

A natural decomposition of $\{0,1, \ldots, n\}$ is $\mathcal{I}_{w_{1}}$, with all parts of width $w_{1}$ (think of $w_{1}$ as a power of 2 that is roughly $\left.n^{1 / 10}\right)$ so $\ell=n / w_{1}$ and $I_{j}=\left\{(j-1) w_{1}, \cdots, j w_{1}\right\}$. An interval of width $w_{1}$ is $\theta$-aligned if its upper and lower endpoints are both multiples of $\theta w_{1}$ (which we require to be an integral power of 2 ). We restrict attention to $x$-intervals in $\mathcal{I}_{w_{1}}$, called $x$-candidates and $\theta$-aligned $y$-intervals of width $w_{1}$ called $y$-candidates. It can be shown (see Proposition 3.4) that an $x$-interval $I$ always has a $\tau$-match $J$ that is $\theta$-aligned. For each $x$-candidate $I$, designate one such $\tau$-match as the canonical $\tau$-match, $J^{\tau}(I)$ for $I$, and $I \times J^{\tau}(I)$ is the canonical $\tau$-compatible box for $I$.

The naïve approach to building $\mathcal{R}$ is to include certified boxes for all choices of $J$ to guarantee a $\tau$-match for each $I_{j}$, i.e., to consider each ( $x$-candidate, $y$-candidate)-pair $(I, J)$, compute its edit distance in time $O\left(w_{1}^{2}\right)$, and include the certified box $\left(I \times J, \Delta_{\text {edit }}(I, J)\right)$ in $\mathcal{R}$. There are $\frac{n}{w_{1}} \cdot \frac{n}{\theta w_{1}}$ boxes, so the time for all edit distance computations is $O\left(\frac{n^{2}}{\theta}\right)$, which is worse than quadratic. (The factor $\frac{1}{\theta}$ can be avoided by standard techniques, but this is not significant to the quest for a subquadratic algorithm, so we defer this until the next section.) Note that $|\mathcal{R}|$ is $\frac{n^{2}}{\theta\left(w_{1}\right)^{2}}$ (which is $n^{1.82}$ for our parameters) so at least the min-cost path phase (which runs in time quasi-linear in $\mathcal{R}$ ) is truly sub-quadratic.

Two natural goals that will improve the running time are: (1) Reduce the amortized time per box needed to certify boxes significantly below $\left(w_{1}\right)^{2}$ and (2) Reduce the total number of certified boxes created significantly below $\frac{n^{2}}{\theta\left(w_{1}\right)^{2}}$. Neither goal is always achievable, and our covering algorithm combines them. In independent work [13, 14], versions of these two goals are combined, where the second goal is accomplished via Grover search, thus yielding a constant factor sub-quadratic time quantum approximation algorithm. (1) builds on ideas from [17] exploiting periodicity of the strings.

Reducing amortized time for certifying boxes: the dense case algorithm. We aim to reduce the amortized time per certified box to be much smaller than $\left(w_{1}\right)^{2}$. We divide our search for certified boxes into iterations $i \in\{0, \ldots, \log n\}$. For iteration $i$, with $\epsilon_{i}=2^{-i}$, our goal is that for all candidate pairs $I, J$ with $\Delta_{\text {edit }}(I, J) \leq \epsilon_{i}$, we include the certified box $\left(I \times J, c \epsilon_{i}\right)$ for a fixed constant $c$. If we succeed, then for each $I_{j}$ and its canonical $\tau$-match $J^{\tau}\left(I_{j}\right)$, and for the largest index $i$ for which $\Delta_{\text {edit }}\left(I_{j}, J^{\tau}\left(I_{j}\right)\right) \leq \epsilon_{i}$, iteration $i$ will certify $\left(I_{j} \times J^{\tau}\left(I_{j}\right), \kappa_{j}\right)$ with $\kappa_{j} \leq c \epsilon_{i} \leq 2 c \Delta_{\text {edit }}\left(I_{j}, J^{\tau}\left(I_{j}\right)\right)$, as needed.

For a string $z$ of size $w_{1}$, let $\mathcal{H}(z, \rho)$ be the set of $x$-candidates $I$ with $\Delta_{\text {edit }}\left(z, x_{I}\right) \leq \rho$ and $\mathcal{V}(z, \rho)$ be the set of $y$-candidates $J$ with $\Delta_{\text {edit }}\left(z, y_{J}\right) \leq \rho$. In iteration $i$, for each $x$-candidate $I$, we will specify a set $\mathcal{Q}_{i}(I)$ of $y$-candidates that includes $\mathcal{V}\left(x_{I}, \epsilon_{i}\right)$ and is contained in $\mathcal{V}\left(x_{I}, 5 \epsilon_{i}\right)$. The set of certified boxes $\left(I \times J, 5 \epsilon_{i}\right)$ for all $x$-candidates $I$ and $J \in \mathcal{Q}_{i}(I)$ satisfies the goal of iteration $i$.

Iteration $i$ proceeds in rounds. At the beginning of the $i$-th iteration we mark all $x$-candidates as 
unfulfilled. In each round we select an $x$-candidate $I$, called the pivot, which is unfulfilled. Compute $\Delta_{\text {edit }}\left(x_{I}, y_{J}\right)$ for all $y$-candidates $J$ and $\Delta_{\text {edit }}\left(x_{I}, x_{I^{\prime}}\right)$ for all $x$-candidates $I^{\prime}$; these determine $\mathcal{H}\left(x_{I}, \rho\right)$ and $\mathcal{V}\left(x_{I}, \rho\right)$ for any $\rho$. For all $I^{\prime} \in \mathcal{H}\left(x_{I}, 2 \epsilon_{i}\right)$, set $\mathcal{Q}_{i}\left(I^{\prime}\right)=\mathcal{V}\left(x_{I}, 3 \epsilon_{i}\right)$. By the triangle inequality, for each $I^{\prime} \in \mathcal{H}\left(x_{I}, 2 \epsilon_{i}\right), \mathcal{V}\left(x_{I}, 3 \epsilon_{i}\right)$ includes $\mathcal{V}\left(x_{I^{\prime}}, \epsilon_{i}\right)$ and is contained in $\mathcal{V}\left(x_{I^{\prime}}, 5 \epsilon_{i}\right)$ so we can certify all the boxes with upper bound $5 \epsilon_{i}$. Mark intervals in $\mathcal{H}\left(x_{I}, 2 \epsilon_{i}\right)$ as fulfilled and proceed to the next round, choosing a new pivot from among the unfulfilled $x$-candidates.

The number of certified boxes produced in a round is $\left|\mathcal{H}\left(x_{I}, 2 \epsilon_{i}\right)\right| \times\left|\mathcal{V}\left(x_{I}, 3 \epsilon_{i}\right)\right|$. If this is much larger than $O\left(\frac{n}{\theta w_{1}}\right)$, the number of edit distance computations, then we have significantly reduced amortized time per certified box. (For example, in the trivial case $i=0$, every candidate box will be certified in a single round.) But in worst case, there are $\frac{n}{w_{1}}$ rounds each requiring $\Omega\left(\frac{n w_{1}}{\theta}\right)$ time, for an unacceptable total time $\Theta\left(n^{2} / \theta\right)$.

Here is a situation where the number of rounds is much less than $\frac{n}{w_{1}}$. Since any two pivots are necessarily greater than $2 \epsilon_{i}$ apart, the sets $\mathcal{V}\left(x_{I}, \epsilon_{i}\right)$ for distinct pivots are disjoint. Now for some parameter $d$ (think of $d=n^{1 / 5}$ ) an $x$-candidate is $d$-dense for $\epsilon_{i}$ if $\left|\mathcal{V}\left(x_{I}, \epsilon_{i}\right)\right| \geq d$, i.e., $x_{I}$ is $\epsilon_{i}$-close in edit distance to at least $d y$-candidates; it is $d$-sparse otherwise. If we manage to select a $d$-dense pivot $I$ in each round, then the number of rounds is $O\left(\frac{n}{w_{1} d \theta}\right)$ and the overall time will be $\Theta\left(\frac{n^{2}}{d \theta^{2}}\right)$. For our parameters this is $\Theta\left(n^{1.84}\right)$. But there's no reason to expect that we'll only choose dense pivots; indeed there need not be any dense pivot.

Let's modify the process a bit. When choosing potential pivot $I$, first test whether or not it is (approximately) $d$-dense. This can be done with high probability, by randomly sampling $\tilde{\Theta}\left(\frac{n}{\theta w_{1} d}\right)$ $y$-candidates and finding the fraction of the sample that are within $\epsilon_{i}$ of $x_{I}$. If this fraction is less than $\frac{\theta w_{1} d}{2 n}$ then $I$ is declared sparse and abandoned as a pivot; otherwise $I$ is declared dense, and used as a pivot. With high probability, all $d$-dense intervals that are tested are declared dense, and all tested intervals that are not $d / 4$-dense are declared sparse, so we assume this is the case. Then all pivots are processed (as above) in time $O\left(\frac{n^{2}}{d \theta^{2}}\right)$ (under our sample parameters: $O\left(n^{1.84}\right)$ ). We pay $\tilde{O}\left(\frac{n}{w_{1} d \theta}\right)\left(w_{1}\right)^{2}$ to test each potential pivot (at most $\frac{n}{w_{1}}$ of them) so the overall time to test potential pivots is $\tilde{O}\left(\frac{n^{2}}{d \theta}\right)$ (with our parameters: $\tilde{O}\left(n^{1.82}\right)$ ).

Each iteration $i$ (with different $\epsilon_{i}$ ) splits $x$-candidates into two sets, $\mathcal{S}_{i}$ of intervals that are declared sparse, and all of the rest for which we have found the desired set $\mathcal{Q}_{i}(I)$. With high probability every interval in $\mathcal{S}_{i}$ is indeed $d$-sparse, but a sparse interval need not belong to $\mathcal{S}_{i}$, since it may belong to $\mathcal{H}\left(x_{I}, 2 \epsilon_{i}\right)$ for some selected pivot $I$.

For every $x$-candidate $I \notin \mathcal{S}_{i}$ we have met the goal for the iteration. If $\mathcal{S}_{i}$ is very small for all iterations (with respect to $\epsilon_{i}$ ), then the set of certified boxes will suffice for the min-cost path algorithm to output a good approximation. But if $\mathcal{S}_{i}$ is not small, another approach is needed.

Reducing the number of candidates explored: the diagonal extension algorithm. For each $x$-candidate $I$, although it suffices to certify the single box $\left(I, J^{\tau}(I)\right)$ with a good upper bound, since $\tau$ is unknown, the exhaustive and dense case approaches both include certified boxes for all $y$-candidates $J$. The potential savings in the dense case approach comes from certifying many boxes simultaneously using a relatively small number of edit distance computations.

Here's another approach: for each $x$-candidate $I$ try to quickly identify a relatively small subset $\mathcal{Y}(I)$ of $y$-candidates that is guaranteed to include $J^{\tau}(I)$. If we succeed, then the number of boxes we certify is significantly reduced, and even paying quadratic time per certified box, we will have a sub-quadratic algorithm.

We need the notion of diagonal extension of a box. The main diagonal of box $I \times J$, is the segment joining the lower left and upper right corners. The square box $I^{\prime} \times J^{\prime}$ is a diagonal extension of a 
square subbox $I \times J$ if the main diagonal of $I \times J$ is a subsegment of the main diagonal of $I^{\prime} \times J^{\prime}$ (see Definition 2). Given square box $I \times J$ and $I \subset I^{\prime}$ the diagonal extension of $I \times J$ with respect to $I^{\prime}$ is the unique diagonal extension of $I \times J$ having $x$-interval $I^{\prime}$. The key observation (Proposition 3.5) is: if $I \times J$ is an adequate cover of $\tau_{I}$ then any diagonal extension $I^{\prime} \times J^{\prime}$ is an adequate cover of $\tau_{I^{\prime}}$.

Now let $w_{1}, w_{2}$ be two numbers with $w_{1} \mid w_{2}$ and $w_{2} \mid n$. (Think of $w_{1}=n^{1 / 10}$ and $w_{2}=n^{3 / 10}$.) We use the decomposition $\mathcal{I}_{w_{2}}$ of $\{0, \ldots, n\}$ into intervals of width $w_{2}$. The set of $y$-candidates consists of $\theta$-aligned vertical intervals of width $w_{2}$ and has size $\frac{n}{\theta w_{2}}$. To identify a small set of potential matches for $I^{\prime} \in \mathcal{I}_{w_{2}}$, we will identify a set (of size much smaller than $\frac{n}{w_{2}}$ ) of $w_{1}$-boxes $\mathcal{B}\left(I^{\prime}\right)$ having $x$-interval in $\mathcal{I}_{w_{1}}\left(I^{\prime}\right)$ (the decomposition of $I^{\prime}$ into width $w_{1}$ intervals). For each box in $\mathcal{B}\left(I^{\prime}\right)$ we determine the diagonal extension $I^{\prime} \times J^{\prime}$ with respect to $I^{\prime}$, compute $\kappa=\Delta_{\text {edit }}\left(I^{\prime}, J^{\prime}\right)$ and certify $\left(I^{\prime} \times J^{\prime}, \kappa\right)$. Our hope is that $\mathcal{B}\left(I^{\prime}\right)$ includes a $\tau$-compatible $w_{1}$-box $I^{\prime \prime} \times J^{\tau}\left(I^{\prime \prime}\right)$. If so then the observation above implies that its diagonal extension provides an adequate cover for $\tau_{I^{\prime}}$.

Here's how to build $\mathcal{B}\left(I^{\prime}\right)$ : Randomly select a $\operatorname{polylog}(n)$ size set $\mathcal{H}\left(I^{\prime}\right)$ of width $w_{1}$ intervals from $\mathcal{I}_{w_{1}}\left(I^{\prime}\right)$. For each $I^{\prime \prime} \in \mathcal{H}\left(I^{\prime}\right)$ compute $\Delta_{\text {edit }}\left(I^{\prime \prime}, J^{\prime \prime}\right)$ for each $y$-candidate $J^{\prime \prime}$, and let $\mathcal{J}\left(I^{\prime \prime}\right)$ consist of the $d$ candidates $J^{\prime \prime}$ with smallest edit distance to $I^{\prime \prime}$. Here $d$ is a parameter; think of $d=n^{1 / 5}$ as before. $\mathcal{B}\left(I^{\prime}\right)$ consists of all $I^{\prime \prime} \times J^{\prime \prime}$ where $I^{\prime \prime} \in \mathcal{H}\left(I^{\prime}\right)$ and $J^{\prime \prime} \in \mathcal{J}\left(I^{\prime \prime}\right)$.

To bound running time: Each $I^{\prime} \in \mathcal{I}_{w_{2}}$ requires $\tilde{O}\left(\frac{n}{\theta w_{1}}\right)$ width- $w_{1} \Delta_{\text {edit }}()$ computations, taking time $\tilde{O}\left(\frac{n w_{1}}{\theta}\right)$. Diagonal extension step requires $\tilde{O}(d)$ width- $w_{2} \Delta_{\text {edit }}()$ computations, for time $\tilde{O}\left(d w_{2}^{2}\right)$. Summing over $\frac{n}{w_{2}}$ choices for $I^{\prime}$ gives time $\tilde{O}\left(n^{2} \frac{w_{1}}{\theta w_{2}}+n d w_{2}\right)$ (with our parameters: $\left.\tilde{O}\left(n^{1.82}\right)\right)$.

Why should $\mathcal{B}\left(I^{\prime}\right)$ include a box that is an adequate approximation to $\tau_{I^{\prime}}$ ? The intuition behind the choice of $\mathcal{B}\left(I^{\prime}\right)$ is that an adequate cover for $\tau_{I^{\prime}}$ should typically be among the cheapest boxes of the form $I^{\prime} \times J^{\prime}$, and if $I^{\prime} \times J^{\prime}$ is cheap then for a randomly chosen $w_{1}$-subinterval $I^{\prime \prime}$, we should also have $I^{\prime \prime} \times J^{\tau}\left(I^{\prime \prime}\right)$ is among the cheapest boxes for $I^{\prime \prime}$.

Clearly this intuition is faulty: $I^{\prime}$ may have many inexpensive matches $J^{\prime}$ such that $I^{\prime} \times J^{\prime}$ is far from $\tau_{I^{\prime}}$, which may all be much cheaper than the match we are looking for. In this bad situation, there are many $y$-intervals $J^{\prime}$ such that $\Delta_{\text {edit }}\left(I^{\prime}, J^{\prime}\right)$ is smaller than the match we are looking for, and this is reminiscent of the good situation for the dense case algorithm, where we hope that $I^{\prime}$ has lots of close matches. This suggests combining the two approaches, and leads to our full covering algorithm.

The full covering algorithm. Given the dense case and diagonal extension algorithms, the full covering algorithm is easy to describe. The parameters $w_{1}, w_{2}, d$ are as above. We iterate over $i \in\{0, \ldots, \log n\}$ with $\epsilon_{i}=2^{-i}$. In iteration $i$, we first run the dense case algorithm, and let $\mathcal{S}_{i}$ be the set of intervals declared sparse. Then run the diagonal extension algorithm described earlier (with small modifications): For each width $w_{2}$ interval $I^{\prime}$, select $\mathcal{H}\left(I^{\prime}\right)=\mathcal{H}_{i}\left(I^{\prime}\right)$ to consist of $\theta\left(\log ^{2} n\right)$ independent random selections from $\mathcal{S}_{i}$. For each $I^{\prime \prime} \in \mathcal{H}_{i}\left(I^{\prime}\right)$, find the set of vertical candidates $J^{\prime \prime}$ for which $\Delta_{\text {edit }}\left(I^{\prime \prime}, J^{\prime \prime}\right) \leq \epsilon_{i}$. Since $I^{\prime \prime}$ is (almost certainly) $d$-sparse, the number of such $J^{\prime \prime}$ is at most $d$. Proceeding as in the diagonal extension algorithm, we produce a set $\mathcal{P}_{i}\left(I^{\prime}\right)$ of $\tilde{O}(d)$ certified $w_{2}$-boxes with $x$-interval $I^{\prime}$. Let $\mathcal{R}_{D}\left(\right.$ resp. $\left.\mathcal{R}_{E}\right)$ be the set of all certified boxes produced by the dense case iterations, resp. diagonal extension iterations. The output is $\mathcal{R}=\mathcal{R}_{D} \cup \mathcal{R}_{E}$. (See Figure 1 for an illustration of the output $\mathcal{R}$.)

The running time is the sum of the running times of the dense case and diagonal extension algorithms, as analyzed above. Later, we will give a more precise analysis of the running time for the pseudo-code. 
To finish this extended overview, we sketch the argument that $\mathcal{R}$ satisfies the covering phase requirements.

Claim 2.1. Let $I^{\prime}$ be an interval in the $w_{2}$-decomposition. Either (1) the output of the dense case algorithm includes a sequence of certified $w_{1}$-boxes that adequately approximates the subpath $\tau_{I^{\prime}}$, or (2) with high probability the output of the sparse case algorithm includes a single $w_{2}$-box that adequately approximates $\tau_{I^{\prime}}$.

(This claim is formalized in Claim [3.12.) Stitching together the subpaths for all $I^{\prime}$ implies that $\mathcal{R}$ contains a sequence of certified boxes that adequately approximates $\tau$.

To prove the claim, we establish a sufficient condition for each of the two conclusions and show that if the sufficient condition for the second conclusion fails, then the sufficient condition for the first holds.

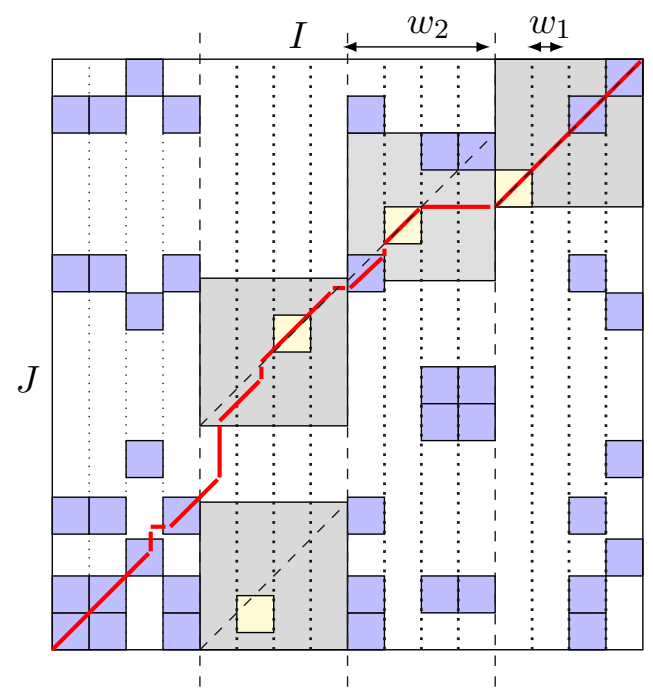

Figure 1: Illustration of the Covering Algorithm: Blue boxes are low cost boxes in dense $w_{1}$-strips, while the yellow ones are in sparse $w_{1}$-strips. The red line corresponds to the path $\tau$ that we are trying to cover. In each $w_{2}$-strip, $\tau$ is covered by either a collection of many $w_{1}$-boxes or it is covered by a diagonal extension of a low cost $w_{1}$-box. The various boxes might overlap vertically which is not shown in the picture.

Let $\mathcal{I}^{\prime}$ denote the $w_{1}$-decomposition $\mathcal{I}_{w_{1}}\left(I^{\prime}\right)$ of $I^{\prime}$. Every interval $I^{\prime \prime} \in \mathcal{I}^{\prime}$ has a $\theta$-aligned $\tau$-match $J^{\tau}\left(I^{\prime \prime}\right)$. It will be shown (see Proposition [3.4), that $\Delta_{\text {edit }}\left(I^{\prime \prime}, J^{\tau}\left(I^{\prime \prime}\right)\right) \leq 2 \frac{\operatorname{cost}\left(\tau_{I^{\prime \prime}}\right)}{\mu\left(I^{\prime \prime}\right)}+\theta$. Let $u\left(I^{\prime \prime}\right)$ denote this upper bound. Consider the first alternative in the claim. During the dense case iteration $i=0$, every interval is declared dense, and $\left(I^{\prime \prime} \times J^{\tau}\left(I^{\prime \prime}\right), 5\right)$ is in $\mathcal{R}_{D}$ for all $I^{\prime \prime}$. To get an adequate approximation, we try to show that later iterations provide much better upper bounds on these boxes, i.e., $\left(I^{\prime \prime} \times J^{\tau}\left(I^{\prime \prime}\right), \gamma\left(I^{\prime \prime}\right)\right) \in \mathcal{R}_{D}$ for a small enough value of $\gamma\left(I^{\prime \prime}\right)$. By definition of adequate approximation, it is enough that $\sum_{I^{\prime \prime} \in \mathcal{I}^{\prime}} \gamma\left(I^{\prime \prime}\right) \leq c \sum_{I^{\prime \prime} \in \mathcal{I}^{\prime}} u\left(I^{\prime \prime}\right)$, for some $c$. Let $t\left(I^{\prime \prime}\right)$ be the last (largest) iteration for which $\epsilon_{t\left(I^{\prime \prime}\right)} \geq u\left(I^{\prime \prime}\right)$ and $I^{\prime \prime} \notin \mathcal{S}_{t\left(I^{\prime \prime}\right)}$ (which is well defined since $\mathcal{S}_{0}=\emptyset$ ). Let 
$b\left(I^{\prime \prime}\right)=\epsilon_{t\left(I^{\prime \prime}\right)}$. Since $b\left(I^{\prime \prime}\right) \geq u\left(I^{\prime \prime}\right) \geq \Delta_{\text {edit }}\left(I^{\prime \prime}, J^{\tau}\left(I^{\prime \prime}\right)\right)$, the box $\left(I^{\prime \prime} \times J^{\tau}\left(I^{\prime \prime}\right), 5 b\left(I^{\prime \prime}\right)\right)$ is certified. The collection $\left\{\left(I^{\prime \prime} \times J^{\tau}\left(I^{\prime \prime}\right), 5 b\left(I^{\prime \prime}\right)\right)\right\}$ is a sequence of certified boxes that satisfies the first two conditions for an adequate approximation of $\tau$. The third condition will follow if:

$$
\sum_{I^{\prime \prime} \in \mathcal{I}^{\prime}} 5 b\left(I^{\prime \prime}\right) \leq c \sum_{I^{\prime \prime} \in \mathcal{I}^{\prime}} u\left(I^{\prime \prime}\right)
$$

so this is sufficient to imply the first condition of the claim.

Next consider what we need for the second alternative to hold. Let $\mathcal{S}_{i}\left(I^{\prime}\right)$ be the set of intervals declared sparse in iteration $i$. An interval $I^{\prime \prime} \in \mathcal{S}_{i}\left(I^{\prime}\right)$ is a winner (for iteration $i$ ) if $\Delta_{\text {edit }}\left(I^{\prime \prime}, J^{\tau}\left(I^{\prime \prime}\right)\right) \leq \epsilon_{i}$, and $\mathcal{W}_{i}\left(I^{\prime}\right)$ is the set of winners. In iteration $i$ of the diagonal extension algorithm, we sample $\theta\left(\log ^{2} n\right)$ elements of $\mathcal{S}_{i}\left(I^{\prime}\right)$. If for at least one iteration $i$ our sample includes a winner $I^{\prime \prime}$ then the second condition of the claim will hold: $I^{\prime \prime} \times J^{\tau}\left(I^{\prime \prime}\right)$ is extended diagonally to a $w_{2}$-box, and by the diagonal extension property, the extension is an adequate cover of $\tau_{I^{\prime}}$, which we will certify with its exact edit distance.

Observe that if there exists $i$ such that $\left|\mathcal{W}_{i}\left(I^{\prime}\right)\right| \geq\left|\mathcal{S}_{i}\left(I^{\prime}\right)-\mathcal{W}_{i}\left(I^{\prime}\right)\right|$ then, except with negligible probability, during the $i$-th iteration our sample includes a winner $I^{\prime \prime}$ (as for each sample the probability of being a winner is at least 1/2). Thus for the second alternative to fail with nonnegligible probability:

$$
\text { For all } i,\left|\mathcal{W}_{i}\left(I^{\prime}\right)\right|<\left|\mathcal{S}_{i}\left(I^{\prime}\right)-\mathcal{W}_{i}\left(I^{\prime}\right)\right| \text {. }
$$

We argue next that if the failure condition (2) holds, then the success condition (1) holds. Multiply (2) by $\epsilon_{i}$ and sum on $i$ to get:

$$
\sum_{I^{\prime \prime} \in \mathcal{I}^{\prime}} \sum_{i: I^{\prime \prime} \in \mathcal{W}_{i}\left(I^{\prime}\right)} \epsilon_{i}<\sum_{I^{\prime \prime} \in \mathcal{I}^{\prime}} \sum_{i: I^{\prime \prime} \in \mathcal{S}_{i}\left(I^{\prime}\right)-\mathcal{W}_{i}\left(I^{\prime}\right)} \epsilon_{i}
$$

For a given interval $I^{\prime \prime} \in \mathcal{I}_{w_{1}}\left(I^{\prime}\right)$, consider the iterations $i$ for which $I^{\prime \prime} \in \mathcal{W}_{i}\left(I^{\prime}\right)$ and those for which $I^{\prime \prime} \in \mathcal{S}_{i}\left(I^{\prime}\right)-\mathcal{W}_{i}\left(I^{\prime}\right)$. First of all if $\epsilon_{i} \geq u\left(I^{\prime \prime}\right)$ and $I^{\prime \prime} \in \mathcal{S}_{i}\left(I^{\prime}\right)$ then since $\Delta_{\text {edit }}\left(I^{\prime \prime}, J^{\tau}\left(I^{\prime \prime}\right)\right) \leq$ $u\left(I^{\prime \prime}\right) \leq \epsilon_{i}$ we conclude $I^{\prime \prime} \in \mathcal{W}_{i}\left(I^{\prime}\right)$. So $I^{\prime \prime} \in \mathcal{S}_{i}\left(I^{\prime}\right)-\mathcal{W}_{i}\left(I^{\prime}\right)$ implies that $\epsilon_{i}<u\left(I^{\prime \prime}\right)$, so the inner sum of the right side of (3) is at most $2 u\left(I^{\prime \prime}\right)$ (by summing a geometric series).

Furthermore, for $i$ with $u\left(I^{\prime \prime}\right) \leq \epsilon_{i}<b\left(I^{\prime \prime}\right), I^{\prime \prime} \in \mathcal{S}_{i}$ by the choice of $t\left(I^{\prime \prime}\right)$. Either $b\left(I^{\prime \prime}\right) / 2 \leq u\left(I^{\prime \prime}\right)$ or $u\left(I^{\prime \prime}\right)<b\left(I^{\prime \prime}\right) / 2$. The latter implies $I^{\prime \prime} \in \mathcal{W}_{t\left(I^{\prime \prime}\right)+1}\left(I^{\prime}\right)$, and then $b\left(I^{\prime \prime}\right) / 2$ is upper bounded by the inner sum on the left of (3). Therefore:

$$
\begin{aligned}
\sum_{I^{\prime \prime}} b\left(I^{\prime \prime}\right) & \leq \sum_{I^{\prime \prime}}\left(2 u\left(I^{\prime \prime}\right)+\sum_{i: I^{\prime \prime} \in \mathcal{W}_{i}\left(I^{\prime}\right)} 2 \epsilon_{i}\right) \\
& <\sum_{I^{\prime \prime}}\left(2 u\left(I^{\prime \prime}\right)+2 \sum_{i: I^{\prime \prime} \in \mathcal{S}_{i}\left(I^{\prime}\right)-\mathcal{W}_{i}\left(I^{\prime}\right)} \epsilon_{i}\right) \\
& \leq 6 \sum_{I^{\prime \prime}} u\left(I^{\prime \prime}\right),
\end{aligned}
$$

as required for (1).

This completes the overview of the covering algorithm. 


\section{Covering Algorithm: pseudo-code and analysis}

The pseudo-code consists of CoveringAlgorithm which calls procedures DenseStripRemoval (the dense case algorithm) and SparseStripExtensionSampling (the diagonal extension algorithm). The technical differences between the pseudo-code and the informal description, are mainly to improve analysis of the running time.

\subsection{Pseudo-code}

The parameters of CoveringAlgorithm are as described in the overview: $x, y$ are input strings of length $n, \theta$ comes from GAP-UB $\mathbf{U B}_{\theta}, w_{1}<w_{2}<n$ and $d<n$ are integral powers of 2 , as are the auxiliary input parameters. The output is a set $\mathcal{R}$ of certified boxes. The algorithm uses global constants $c_{0} \geq 0$ and $c_{1} \geq 640$, where the former one is needed for Proposition 3.8.

We use a subroutine SMALL-ED which takes strings $z_{1}, z_{2}$ of length $w$ and parameter $\kappa$ and outputs $\infty$ if $\Delta_{\text {edit }}\left(z_{1}, z_{2}\right)>\kappa$ and otherwise outputs $\Delta_{\text {edit }}\left(z_{1}, z_{2}\right)$. The algorithm of [26] implements SMALL-ED in time $O\left(\kappa w^{2}\right)$.

One technical difference from the overview, is that the pseudo-code saves time by restricting the search for certified boxes to a portion of the grid close to the main diagonal. Recall that GAP-UB $\mathbf{B}_{\theta}$ has two requirements, that the output upper bounds $d_{\text {edit }}(x, y)$ (which will be guaranteed by the requirement that $\mathcal{R}$ contains no falsely certified boxes), and that if $d_{\text {edit }}(x, y) \leq \theta n$, the output is at most $c \theta n$ for some constant $c$. We therefore design our algorithm assuming $d_{\text {edit }}(x, y) \leq \theta n$, in which case every min-cost $G_{x, y}$-path $\tau$ consists entirely of points $(i, j)$ within $\frac{\theta}{2} n$ steps from the main diagonal, i.e. $|i-j| \leq \frac{\theta}{2} n$. So we restrict our search for certified boxes as follows: set $m=\frac{1}{4} \theta n$, and consider the $\frac{n}{m}$ overlapping equally spaced boxes of width $8 m=2 \theta n$ lying along the main diagonal. Together these boxes cover all points within $\theta n$ of the main diagonal.

The algorithm of the overview is executed separately on each of these $n / m$ boxes. Within each of these executions, we iterate over $i \in\left\{0, \ldots, \log \frac{1}{\theta}\right\}$ (rather than $\{0, \ldots, \log n\}$ as in the overview). In each iteration we apply the dense case algorithm and the diagonal extension algorithm as in the overview. The output is the union over all $n / m$ boxes and all iterations, of the boxes produced.

In the procedures DenseStripRemoval and SparseStripExtensionSampling, the input $G$ is an induced grid graph corresponding to a box $I_{G} \times J_{G}$, as described in the "framework" part of Section 1. The procedure DenseStripRemoval on input $G$, sets $\mathcal{T}$ to be the $w_{1}$-decomposition of $I_{G}$ (the $x$-candidates) and $\mathcal{B}$ to be the set of $\frac{\epsilon_{i}}{8}$-aligned $y$-candidates. As in the overview, the dense case algorithm produces a set of certified boxes (called $\mathcal{R}_{1}$ in the pseudo-code) and a set $\mathcal{S}$ of intervals declared sparse. SparseStripExtensionSampling is invoked if $\mathcal{S} \neq \emptyset$ and iterates over all $x$-intervals $I^{\prime}$ in the decomposition $\mathcal{I}_{w_{2}}\left(I_{G}\right)$. The algorithm skips $I^{\prime}$ if $\mathcal{S}$ contains no subset of $I^{\prime}$, and otherwise selects a sample $\mathcal{H}$ of $\theta\left(\log ^{2} n\right)$ subintervals of $I^{\prime}$ from $\mathcal{S}$. For each sample interval $I^{\prime \prime}$ it finds the vertical candidates $J^{\prime \prime}$ for which $\Delta_{\text {edit }}\left(I^{\prime \prime}, J^{\prime \prime}\right) \leq \epsilon_{i}$, does a diagonal extension to $I^{\prime}$ and certifies each box with an exact edit distance computation.

There are a few parameter changes from the overview that provide some improvement in the time analysis: During each iteration $i$, rather than taking our vertical candidates to be from a $\theta$-aligned grid, we can afford a grid that is $\epsilon_{i} / 8$-aligned which is coarser for $\epsilon_{i} \gg \theta$. Also, the local parameter $d$ in DenseStripRemoval and SparseStripExtensionSampling is set to $d / \epsilon_{i}$ during iteration $i$.

There is one counterintuitive quirk in SparseStripExtensionSampling: each certified box is replicated $O(\log n)$ times with higher distance bounds. This is permissible (increasing the distance bound cannot decertify a box), but seems silly (why add the same box with a higher distance bound?). 
This is just a convenient technical device to ensure that the second phase min-cost path algorithm gives a good approximation (using adjacent boxes that overlap vertically).

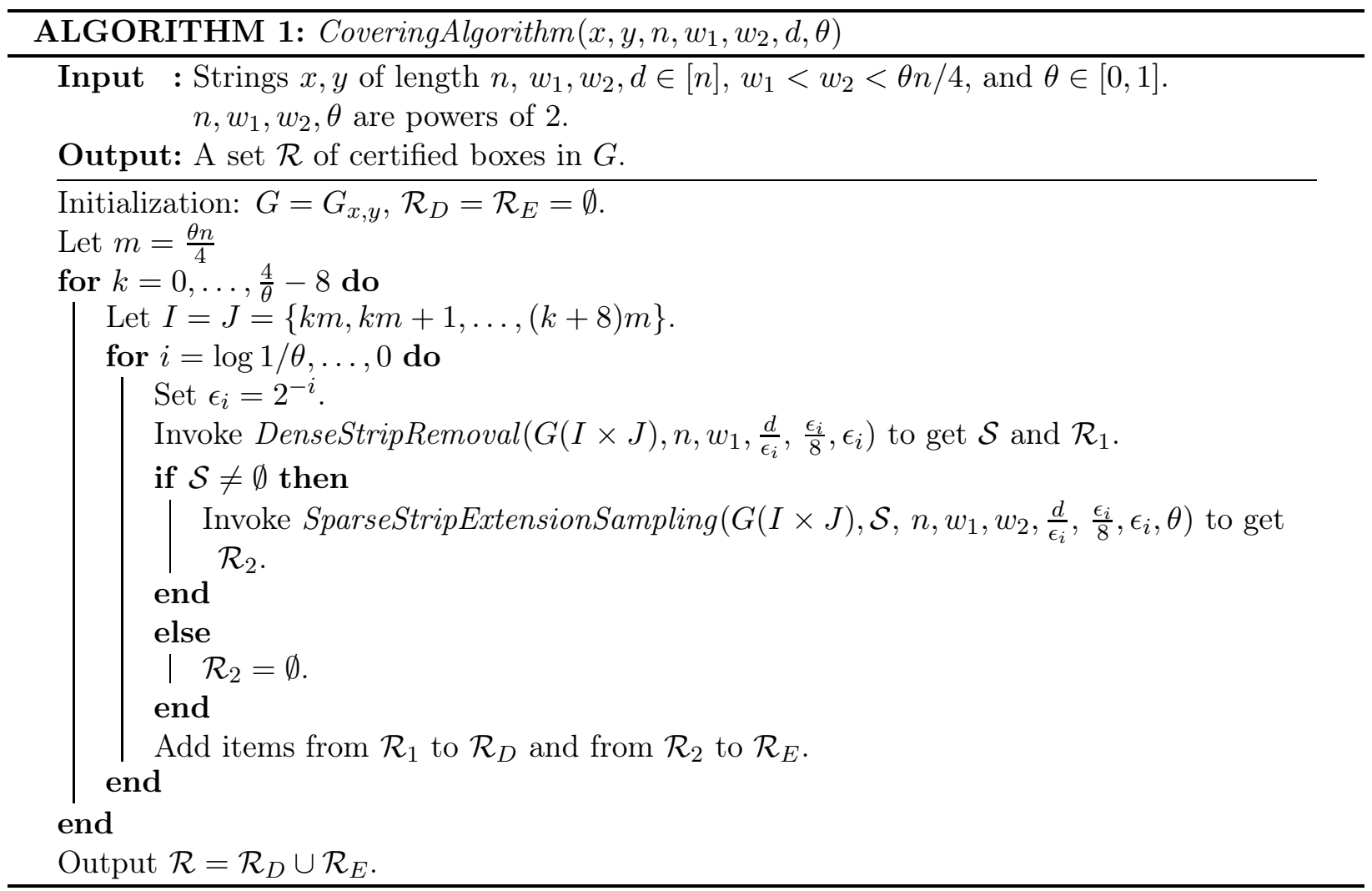

For the analysis we must prove that $\mathcal{R}$ contains an "adequate approximation" of some min-cost alignment path $\tau$. To state this precisely, we start with definitions and observations that formalize intuitive notions from the overview.

Cost and normalized cost. The cost of a path $\tau, \operatorname{cost}(\tau)$, from $\left(u_{1}, u_{2}\right)$ to $\left(v_{1}, v_{2}\right)$ in a gridgraph (see Section 1), is the sum of the edge costs, and the normalized cost is $\operatorname{ncost}(\tau)=\frac{\operatorname{cost}(\tau)}{v_{1}-u_{1}}$. $\operatorname{cost}(G(I \times J))$ (or simply cost $(I \times J))$, the cost of subgraph $G(I \times J)$, is the min-cost of a path from the lower left to the upper right corner. The normalized cost is $\operatorname{ncost}(I \times J)=\frac{1}{\mu(I)} \cdot \operatorname{cost}(I \times J)$.

We note the following simple fact without proof:

Proposition 3.1. For $I, J, J^{\prime} \subseteq\{0, \ldots, n\},\left|\operatorname{cost}(I \times J)-\operatorname{cost}\left(I \times J^{\prime}\right)\right| \leq\left|J \Delta J^{\prime}\right|$, where $\Delta$ denotes symmetric difference.

Projections and subpaths. The horizontal projection of a path $\tau=\left(i_{1}, j_{1}\right), \ldots,\left(i_{\ell}, j_{\ell}\right)$ is the set of $\left\{i_{1}, \ldots, i_{\ell}\right\}$. We say that $\tau$ crosses box $I \times J$ if the vertices of $\tau$ belong to $I \times J$ and its horizontal projection is $I$. If the horizontal projection of $\tau$ contains $I^{\prime}, \tau_{I^{\prime}}$ denotes the (unique) minimal subpath of $\tau$ whose projection is $I^{\prime}$.

Proposition 3.2. Let $\tau$ be a path with horizontal projection $I$, and let $I_{1}, \ldots, I_{\ell}$ be a decomposition of $I$. Then the $\tau_{I_{j}}$ are edge-disjoint and so: 


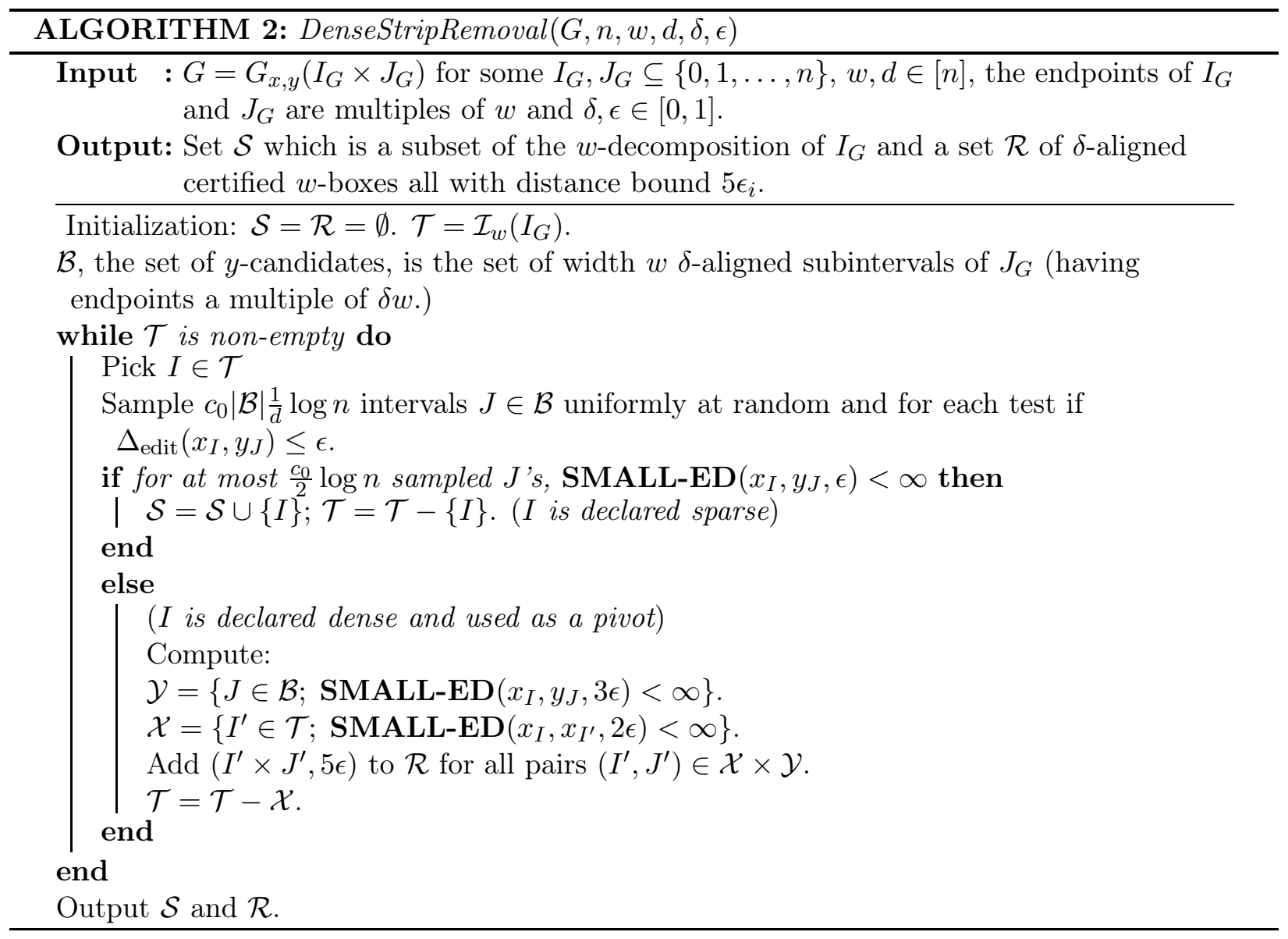




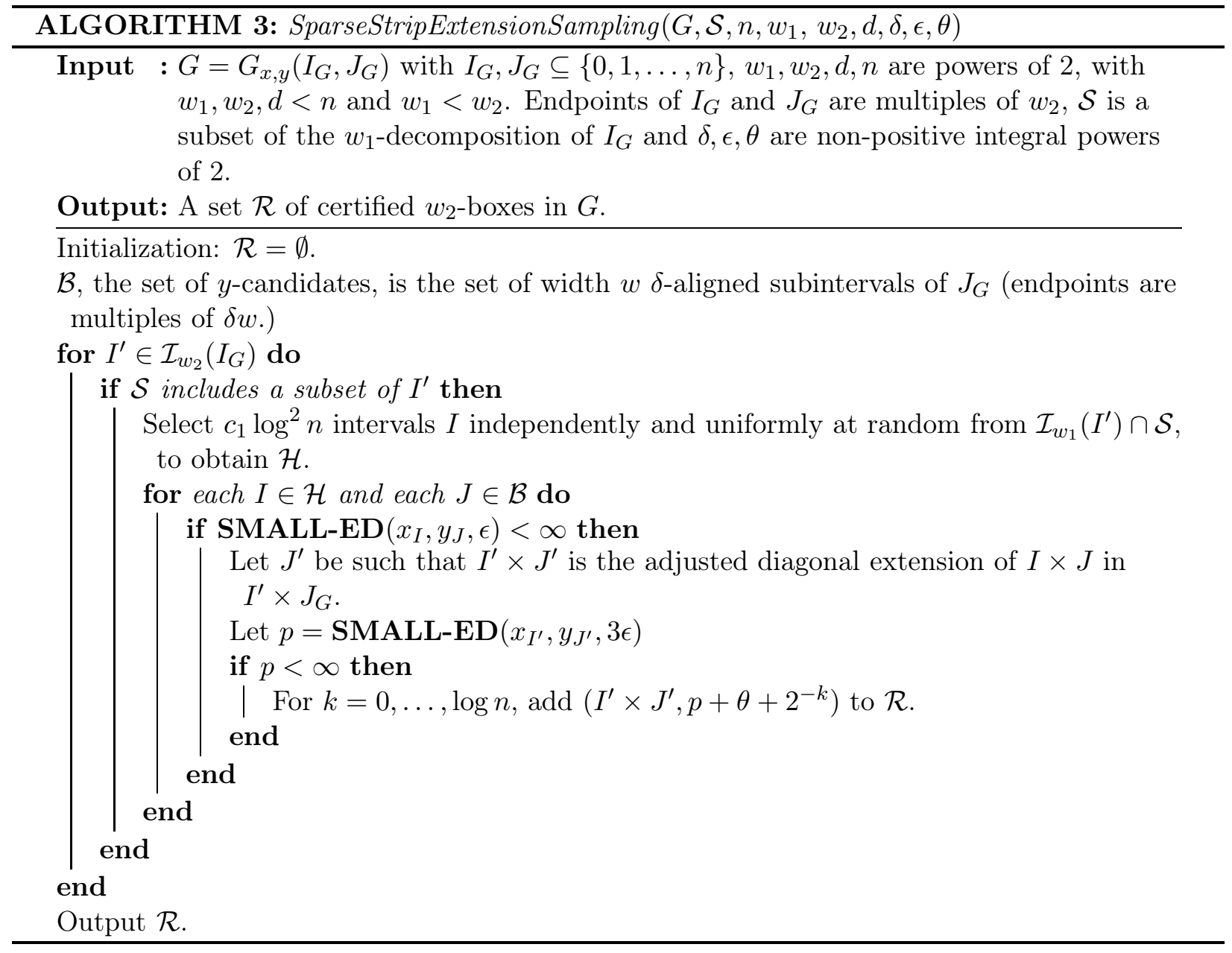




$$
\begin{aligned}
\operatorname{cost}(\tau) & \geq \sum_{i=1}^{\ell} \operatorname{cost}\left(\tau_{I_{i}}\right) \\
n \operatorname{cost}(\tau) & \geq \sum_{i=1}^{\ell} \frac{\mu\left(I_{i}\right)}{\mu(I)} n \operatorname{cost}\left(\tau_{I_{i}}\right) .
\end{aligned}
$$

Definition 1. $(1-\delta)$-cover. Let $\tau$ be a path with horizontal projection $I$ and let $I^{\prime} \times J^{\prime}$ be $a$ (not necessarily square) box with $I^{\prime} \subseteq I$. For $\delta \geq 0$ the box $I^{\prime} \times J^{\prime}(1-\delta)$-covers $\tau$ if the initial, resp. final, vertex of the subpath $\tau_{I^{\prime}}$ is within $\delta \mu\left(I^{\prime}\right)$ vertical units of $\left(\min \left(I^{\prime}\right), \min \left(J^{\prime}\right)\right)$, resp. $\left(\max \left(I^{\prime}\right), \max \left(J^{\prime}\right)\right)$.

We will be interested in $(1-\delta)$-cover when $\delta \leq 1 / 2$ but for simplicity we allow $\delta>1 / 2$.

Proposition 3.3. Let $I^{\prime} \times J^{\prime}$ be a (not necessarily square) box that $(1-\delta)$-covers path $\tau$.

1. $n \operatorname{cost}\left(I^{\prime} \times J^{\prime}\right) \leq n \operatorname{cost}\left(\tau_{I^{\prime}}\right)+2 \delta$.

2. If $J^{\prime \prime}$ is any vertical interval such that $J^{\prime} \cap J^{\prime \prime} \neq \emptyset$, then $I^{\prime} \times J^{\prime \prime}\left(1-\delta-\left|J^{\prime} \Delta J^{\prime \prime}\right| / \mu\left(I^{\prime}\right)\right)$-covers $\tau$.

Proof. For the first part, let $J^{0}$ be the vertical projection of $\tau_{I^{\prime}}$. Then $\operatorname{ncost}\left(I^{\prime} \times J^{0}\right) \leq \operatorname{ncost}\left(\tau_{I^{\prime}}\right)$ since $\tau_{I^{\prime}}$ joins the lower left corner of $I^{\prime} \times J^{0}$ to the upper right corner. Since $I^{\prime} \times J^{\prime}(1-\delta)$-covers $\tau,\left|J^{\prime} \Delta J^{0}\right| \leq 2 \delta \mu\left(I^{\prime}\right)$, and by Proposition 3.1, ncost $\left(I^{\prime} \times J^{\prime}\right) \leq \operatorname{ncost}\left(\tau_{I^{\prime}}\right)+2 \delta$.

For the second part, observe that the vertical distance between the lower (resp. upper) corners of $I^{\prime} \times J^{\prime}$ and $I^{\prime} \times J^{\prime \prime}$ is at most $\left|J^{\prime} \Delta J^{\prime \prime}\right|$.

$\delta$-aligned boxes. A $y$-interval $J$ of width $w$ is $\delta$-aligned for $\delta \in(0,1]$ if its endpoints are multiples of $\delta w$ (which we require to be an integer).

Proposition 3.4. Let $\tau$ be a path that crosses $I \times J$. Suppose that $I^{\prime} \subseteq I$ has width $w$, and $\mu(J) \geq w$.

1. There is an interval $J^{1}$ with $\mu\left(J^{1}\right)=\mu\left(I^{\prime}\right)$ so that $n \operatorname{cost}\left(I^{\prime} \times J^{1}\right) \leq 2 n \operatorname{cost}\left(\tau_{I^{\prime}}\right)$ and $I^{\prime} \times J^{1}$ $\left(1-n \operatorname{cost}\left(\tau_{I^{\prime}}\right)\right)$-covers $\tau$.

2. There is a $\delta$-aligned interval $J^{\prime}$ of width $w$ so that $n \operatorname{cost}\left(I^{\prime} \times J^{\prime}\right) \leq 2 n \operatorname{cost}\left(\tau_{I^{\prime}}\right)+\delta$ and $I^{\prime} \times J^{\prime}$ $\left(1-n \operatorname{cost}\left(\tau_{I^{\prime}}\right)-\delta\right)$-covers $\tau$. Moreover, if $J$ is $\delta$-aligned and $w \mid \mu(J)$ then $J^{\prime} \subseteq J$.

$\left(J^{1}, J^{\prime}\right.$ are " $\tau$-matches" for $I^{\prime}$, in the sense of the overview.)

Proof. Let $\tau^{\prime}=\tau_{I^{\prime}}$ be the min-cost subpath of $\tau$ that projects to $I^{\prime}$. Let $J^{0}$ be the vertical projection of $\tau^{\prime}$. Note that $\left|\mu\left(J^{0}\right)-\mu\left(I^{\prime}\right)\right| \leq \operatorname{cost}\left(\tau^{\prime}\right)$. Arbitrarily choose an interval $J^{1}$ of width $\mu\left(I^{\prime}\right)$ that either contains or is contained in $J^{0}$. Then $\left|J^{0} \Delta J^{1}\right|=\left|\mu\left(J^{0}\right)-\mu\left(I^{\prime}\right)\right| \leq \operatorname{cost}\left(\tau^{\prime}\right)$, so by Proposition $3.1 \mathrm{ncost}\left(I^{\prime} \times J^{1}\right) \leq 2 \mathrm{ncost}\left(\tau^{\prime}\right)$. Furthermore $I^{\prime} \times J^{1}\left(1-\operatorname{ncost}\left(\tau^{\prime}\right)\right)$-covers $\tau^{\prime}$. Let $J^{\prime}$ be the closest $\delta$ aligned interval to $J^{1}$, so $\left|J^{\prime} \Delta J^{1}\right| \leq \delta \mu\left(I^{\prime}\right)$ and so $\operatorname{ncost}\left(I^{\prime} \times J^{\prime}\right) \leq \operatorname{ncost}\left(I^{\prime} \times J^{1}\right)+\delta \leq 2 \operatorname{ncost}\left(\tau^{\prime}\right)+\delta$. Finally since $I^{\prime} \times J^{\prime}$ is a vertical shift of $I^{\prime} \times J^{1}$ of normalized length at most $\delta$, we have $I^{\prime} \times J^{\prime}$ $\left(1-\operatorname{ncost}\left(\tau^{\prime}\right)-\delta\right)$-covers $\tau^{\prime}$. 
Definition 2. 1. The main diagonal of a box is the segment joining the lower left and upper right corners.

2. For a square box $I^{\prime} \times J^{\prime}$, and $I^{\prime} \subseteq I$, the true diagonal extension of $I^{\prime} \times J^{\prime}$ to $I$ is the square box $I \times \hat{J}$ whose main diagonal contains the main diagonal of $I^{\prime} \times J^{\prime}$ (in the infinite grid graph on $\mathbb{Z} \times \mathbb{Z})$.

3. For a $w$-box $I^{\prime} \times J^{\prime}$ contained in strip $I \times J$, the adjusted diagonal extension of $I^{\prime} \times J^{\prime}$ within $I \times J$ is the box $I \times J^{\prime \prime}$ obtained from the true diagonal extension of $I^{\prime} \times J^{\prime}$ to $I$ by the minimal vertical shift so that it is a subset of $I \times J$. (The adjusted diagonal extension is the true diagonal extension if the true diagonal extension is contained in $I \times J$; otherwise it's lower edge is $\min (J)$ or its upper edge is $\max (J)$.)

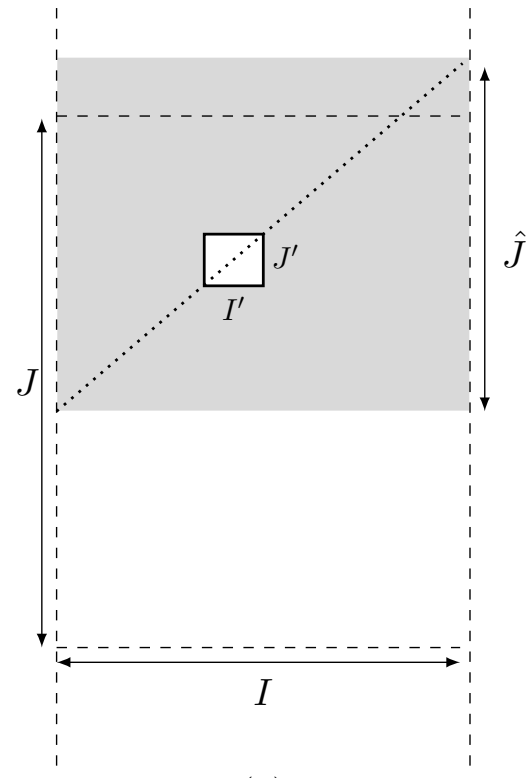

(a)

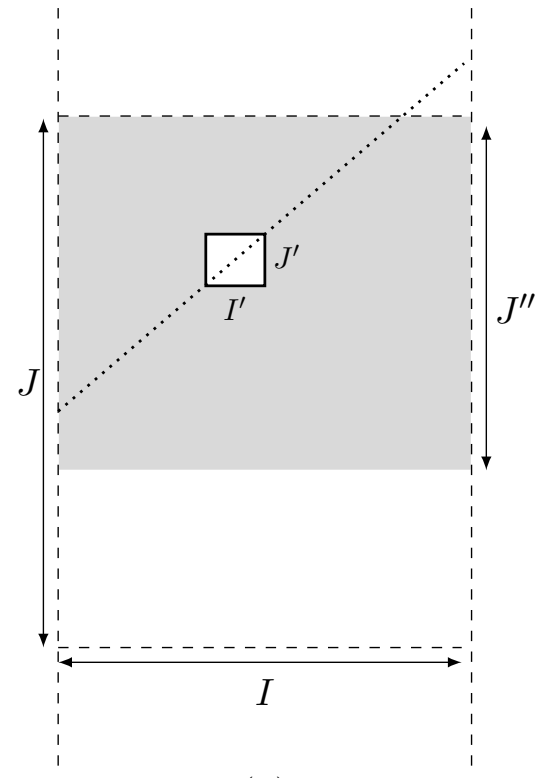

(b)

Figure 2: Illustration of diagonal extension: Given a square box $I^{\prime} \times J^{\prime}$, the true diagonal extension is the grey box $I \times \hat{J}$ in $(a)$ and the adjusted diagonal extension is the grey box $I \times J^{\prime \prime}$ in $(b)$.

Proposition 3.5. Suppose path $\tau$ crosses $I \times J$ and $n \operatorname{cost}\left(\tau_{I}\right) \leq \epsilon$. Let $w=\mu(I)$. Let $I^{\prime} \times J^{\prime}$ be a $w^{\prime}$-box that $(1-\delta)$-covers $\tau_{I^{\prime}}$ and $I^{\prime} \subseteq I$. Then the adjusted diagonal extension $I \times J^{\prime \prime}$ of $I^{\prime} \times J^{\prime}$ within $I \times J\left(1-\left(\epsilon+\delta \frac{w^{\prime}}{w}\right)\right)$-covers $\tau$ and satisfies $n \operatorname{cost}\left(I \times J^{\prime \prime}\right) \leq 3 \epsilon+2 \delta \frac{w^{\prime}}{w}$.

Proof. It suffices to show that $I \times J^{\prime \prime}\left(1-\left(\epsilon+\delta w^{\prime} / w\right)\right)$-covers $\tau$, since then Proposition 3.3 gives us the needed upper bound on $\operatorname{ncost}\left(I \times J^{\prime \prime}\right)$.

Case 1. $I \times J^{\prime \prime}$ is equal to the true diagonal extension. Let $\tau_{I}, \tau_{I^{\prime}}$ be the min-cost subpath of $\tau$ that projects on $I$ and $I^{\prime}$ respectively.

We will give an upper bound on the vertical distance from the final vertex of $\tau_{I}$ to the upper right corner of $I \times J^{\prime \prime}$. Let $\tau_{u}$ be the subpath of $\tau$ that starts at the final vertex of $\tau_{I^{\prime}}$ and ends 
at the final vertex of $\tau_{I}$. Let $I_{u}$ and $J_{u}$ be the horizontal and vertical projections of $\tau_{u}$. The start vertex of $\tau_{u}$ has vertical distance at most $\delta w^{\prime}$ from the main diagonal of $I \times J^{\prime \prime}$. The final vertex of $\tau_{u}$ therefore has vertical distance at most $\delta w^{\prime}+\left|\mu\left(I_{u}\right)-\mu\left(J_{u}\right)\right|$ from the upper corner of $I \times J^{\prime \prime}$, and this is at most $\delta w^{\prime}+\epsilon w$, since $\operatorname{cost}(\tau) \geq\left|\mu\left(I_{u}\right)-\mu\left(J_{u}\right)\right|$. A similar argument gives the same upper bound on the vertical distance between the start vertex of $\tau_{I}$ and the lower left corner of $I \times J^{\prime \prime}$, so $G^{\prime \prime}\left(I \times J^{\prime \prime}\right)\left(1-\left(\epsilon+\delta w^{\prime} / w\right)\right)$-covers $\tau$.

Case 2. $I \times J^{\prime \prime}$ is not the true diagonal extension. Extend the set $J$ to $\bar{J}$ by adding $\mu(I)$ elements before and after. (It is possible that $\bar{J}$ is not a subset of $\{0, \ldots, n\}$; in this case we imagine that $y$ is extended to a sequence $y^{*}$ by adding $\mu(I)$ new symbols to the beginning and end of $y$ and that we are in the grid graph $G_{x, y^{*}}$.) Let $I \times J^{\prime \prime \prime}$ be the adjusted diagonal extension of $I^{\prime} \times J^{\prime}$ to $I \times \bar{J}$. This is equal to the true diagonal extension, and so by Case $1, I \times J^{\prime \prime \prime}\left(1-\left(\epsilon+\delta w^{\prime} / w\right)\right)$-covers $\tau$. We claim that $I \times J^{\prime \prime}$ does also. Assume $J^{\prime \prime \prime}$ falls below $\min (J)$ (the case that $J^{\prime \prime \prime}$ is above $\max (J)$ is similar). Then $I \times J^{\prime \prime}$ is obtained by shifting $I \times J^{\prime \prime \prime}$ up until the lower edge coincides with $\min (J)$. The lower vertex of $\tau_{I}$ has $y$-coordinate at least $\min (J)$.

If the $y$-coordinate of the upper vertex of $\tau_{I}$ is at most $\max \left(J^{\prime \prime}\right)$, then $J^{\prime \prime}$ contains vertical projection of $\tau_{I}$, and $I \times J^{\prime \prime}(1-\epsilon)$-covers $\tau$. If the $y$-coordinate of the upper vertex of $\tau_{I}$ is greater than $\max \left(J^{\prime \prime}\right)$, shifting $I \times J^{\prime \prime \prime}$ up to $I \times J^{\prime \prime}$ can only decrease the vertical distance from the the lower left corner to the start of $\tau_{I}$ and from the upper corner to the end of $\tau_{I}$, so $I \times J^{\prime \prime}$ $\left(1-\left(\epsilon+\delta w^{\prime} / w\right)\right)$-covers $\tau$.

$(k, \zeta)$-approximation of a path. This formalizes the notion of adequate approximation of a path by a certified box sequence.

Definition 3. Let $G$ be the grid graph on $I \times J$. Let $\zeta \in[0,1]$. Let $\tau$ be a path that crosses $G$. $A$ sequence of certified boxes $\sigma=\left\{\left(I_{1} \times J_{1}, \epsilon_{1}\right),\left(I_{2} \times J_{2}, \epsilon_{2}\right), \ldots,\left(I_{\ell} \times J_{\ell}, \epsilon_{\ell}\right)\right\}(k, \zeta)$-approximates $\tau$ provided that:

1. $I_{1}, \ldots, I_{\ell}$ is a decomposition of $I$.

2. For each $i \in[\ell], I_{i} \times J_{i}\left(1-\epsilon_{i}\right)$-covers $\tau$.

3. $\sum_{i \in[\ell]} \epsilon_{i} \mu\left(I_{i}\right) \leq(k \cdot n \operatorname{cost}(\tau)+\zeta) \mu(I)$.

Proposition 3.6. Suppose path $\tau$ crosses $I \times J$ and $I_{1}, \ldots, I_{m}$ is a decomposition of $I$, and for $i \in$ $[m], \sigma_{i}$ is a certified box sequence that $(k, \zeta)$-approximates $\tau_{I_{i}}$. Then $\sigma_{1}, \ldots, \sigma_{m}(k, \zeta)$-approximates $\tau$.

Proof. It is obvious that $\sigma_{1}, \ldots, \sigma_{m}$ is a sequence of certified boxes, that the horizontal projections of all the boxes form a decomposition of $I$ and that each box $\left(I_{i}^{\prime}, J_{i}^{\prime}, \epsilon_{i}\right)\left(1-\epsilon_{i}\right)$-covers $\tau$. The final condition is verified by splitting the sum on the left into $m$ sums where the $j$ th sum includes terms for $I_{i}^{\prime} \subseteq I_{j}$, and is bounded above by $\left(k \cdot \operatorname{ncost}\left(\tau_{I_{j}}\right)+\zeta\right) \mu\left(I_{j}\right)$. Summing the latter sum over $j$ and using Proposition 3.2 we get that $\sigma_{1}, \ldots, \sigma_{m}(k, \zeta)$-approximates the path $\tau$.

$(d, \delta, \epsilon)$-dense and -sparse. Fix a box $I \times J$. An interval $I^{\prime} \subseteq I$ of width $w$ is $(d, \delta, \epsilon)$-sparse (wrt $I \times J)$ for integer $d$ and $\epsilon, \delta \in(0,1]$ if there are at most $d \delta$-aligned $w$-boxes in $I^{\prime} \times J$ of ncost at most $\epsilon$, and is $(d, \delta, \epsilon)$-dense otherwise.

The sets $\mathcal{S}_{i}$ and $\mathcal{S}_{i}\left(I^{\prime}\right)$. For fixed $k$ in the outer loop of CoveringAlgorithm, the set $\mathcal{S}$ created in iteration $i$ of CoveringAlgorithm is denoted by $\mathcal{S}_{i}$. For any interval $I^{\prime}, \mathcal{S}_{i}\left(I^{\prime}\right)$ is the set of subintervals of $I^{\prime}$ belonging to $\mathcal{S}_{i}$. 
Successful Sampling. The algorithm uses random sampling in two places: in DenseStripRemoval to test density of width $w_{1}$ intervals $I$, and in SparseStripExtensionSampling to sample sparse intervals $I \in \mathcal{I}_{w_{1}}\left(I^{\prime}\right)$.

We now specify what we need from the random sampling.

Definition 4. We define two events where the latter depends on the former:

- A run of the algorithm has successful classification sampling provided that for all $k \in\{0, \ldots$, $4 / \theta-8\}$ and $i \in\left\{0, \ldots, \log \frac{1}{\theta}\right\}$ in the nested CoveringAlgorithm loops, in each call to DenseStripRemoval(), for every width $w_{1}$ interval I with endpoints a multiple of $w_{1}$, if I is $\left(\frac{d}{\epsilon_{i}}, \frac{\epsilon_{i}}{8}, \epsilon_{i}\right)$ dense interval (in terms of global CoveringAlgorithm's parameters with respect to CoveringAlgorithm's $I \times J)$, DenseStripRemoval does not assign $I$ to $\mathcal{S}$ and if $I$ is $\left(\frac{d}{4 \epsilon_{i}}, \frac{\epsilon_{i}}{8}, \epsilon_{i}\right)$-sparse and DenseStripRemoval() picks it from $\mathcal{T}$, DenseStripRemoval places $I$ in $\mathcal{S}$.

- Let $\tau$ be a source-sink path in $G_{x, y}$. For each $I^{\prime} \in \mathcal{I}_{w_{2}}$, for $I=J=\{k m, k m+1, \ldots,(k+$ 8) $m\}$, where $k \in\left\{0, \ldots, \frac{4}{\theta}-8\right\}$ is the largest integer such that $k m \leq \min \left(I^{\prime}\right)-\frac{\theta n}{2}$ or 0 if $\min \left(I^{\prime}\right)<\frac{\theta n}{2}$, define set $\mathcal{S}_{i}\left(I^{\prime}\right)$ to be $\mathcal{S} \cap \mathcal{I}_{w_{1}}\left(I^{\prime}\right)$ where $\mathcal{S}$ is returned by the invocation of DenseStripRemoval $\left(G(I \times J), n, w_{1}, \frac{d}{\epsilon_{i}}, \frac{\epsilon_{i}}{8}, \epsilon_{i}\right)$. Furthermore, define the set of winners $\mathcal{W}_{i}\left(I^{\prime}\right)=$ $\left\{I^{\prime \prime} \in \mathcal{S}_{i}\left(I^{\prime}\right), \epsilon_{i} \geq 3 n \operatorname{cost}\left(\tau_{I^{\prime \prime}}\right)+n \operatorname{cost}\left(\tau_{I^{\prime}}\right)+\theta\right\}$.

We say a run of the algorithm has successful extension sampling for $\tau$ provided that: for each $i \in\left\{0, \ldots, \log \frac{1}{\theta}\right\}$ and $I^{\prime} \in \mathcal{I}_{w_{2}}$, if $\left|\mathcal{W}_{i}\left(I^{\prime}\right)\right| \geq \frac{1}{64}\left|\mathcal{S}_{i}\left(I^{\prime}\right)\right|$ and both are non-empty then at least one interval from $\mathcal{W}_{i}\left(I^{\prime}\right)$ will be included in the set $\mathcal{H}$ sampled in iteration $I^{\prime}$ of the outer loop during invocation of SparseStripExtensionSampling $\left(G(I \times J), \mathcal{S}, n, w_{1}, w_{2}, \frac{d}{\epsilon_{i}}, \frac{\epsilon_{i}}{8}, \epsilon_{i}, \theta\right)$.

We say a run of the algorithm has successful sampling for $\tau$ if it has successful classification sampling and successful extension sampling for $\tau$.

We will need the following variant of the Chernoff bound.

Proposition 3.7 (Chernoff bound). There is a constant $c_{0}$ such that the following is true. Let $1 \leq d \leq n$ be integers, $B$ be a set and $E \subseteq B$. Let us sample $c_{0} \frac{|B|}{d} \log n$ samples from $B$ independently at random with replacement.

1. If $|E| \geq d$ then the probability that less than $\frac{c_{0}}{2} \log n$ samples are from $E$ is at most $1 / n^{10}$.

2. If $|E| \leq d / 4$ then the probability that at least $\frac{c_{0}}{2} \log n$ samples are from $E$ is at most $1 / n^{10}$.

Proposition 3.8. For large enough $n$ and any source-sink path in $G_{x, y}$, a run of CoveringAlgorithm has successful sampling for $\tau$ with probability at least $1-n^{-7}$.

Proof. By Proposition 3.7, the probability that the condition of successful classification sampling fails for a particular $k, i, I$ is at most $n^{-10}$. The number of choices for $k, i, I$ is at most $\frac{4}{\theta} \cdot(1+$ $\left.\log \frac{1}{\theta}\right) \frac{n}{w_{1}} \leq n^{2}$ (for large enough $n$ ) so the overall probability that (1) fails is at most $n^{-8}$.

The probability that the condition of successful extension sampling for $\tau$ fails for a particular $i, I^{\prime}$ is $\left(1-\frac{1}{64}\right)^{c_{1} \log ^{2} n} \leq n^{-10}$. The number of $i, I^{\prime}$ is less than $n^{2}$ (for large enough $n$ ), so the overall failure probability is at most $n^{-8}$.

We assume that coins are fixed in a way that gives successful sampling for some shortest sourcesink path in $G_{x, y}$. 


\subsection{Properties of the covering algorithm}

The main property of CoveringAlgorithm to be proved is:

Theorem 3.9. Let $x, y$ be strings of length $n, 1 / n \leq \theta \leq 1$ be a real. Let $w_{1}, w_{2}$, d satisfy $w_{1} \leq \theta w_{2}$, $w_{2} \leq \frac{\theta n}{4}$ and $1 \leq d \leq \frac{\theta n}{w_{1}}$. Assume $n, w_{1}, w_{2}, d, \theta$ are powers of 2 . Let $\mathcal{R}$ be the set of weighted boxes obtained by running CoveringAlgorithm $\left(x, y, n, w_{1}, w_{2}, d, \theta\right)$ with $c_{1}>640$. Then

1. Every $(I \times J, \epsilon) \in \mathcal{R}$ is correctly certified, i.e., $\Delta_{\text {edit }}\left(x_{I}, y_{J}\right) \leq \epsilon$, and

2. For every source-sink path $\tau$ in $G=G_{x, y}$ of normalized cost at most $\theta$, in a run that satisfies successful sampling for $\tau$, there is a subset of $\mathcal{R}$ that $(45,15 \theta)$-approximates $\tau$.

Proof. All boxes output are correctly certified: Each box in $\mathcal{R}_{E}$ comes from SparseStripExtensionSampling which only certifies boxes with at least their exact edit distance. For $(I \times J, \epsilon) \in \mathcal{R}_{D}$, there must be an $I^{\prime}$ such that $\Delta_{\text {edit }}\left(x_{I^{\prime}}, y_{J}\right) \leq \frac{3}{5} \cdot \epsilon$ and $\Delta_{\text {edit }}\left(x_{I^{\prime}}, x_{I}\right) \leq \frac{2}{5} \cdot \epsilon$ and so by triangle inequality $\Delta_{\text {edit }}\left(x_{I}, y_{J}\right) \leq \epsilon$.

It remains to establish (2). Fix a source-sink path $\tau$ of normalized cost $\leq \theta$. By Proposition 3.6 it is enough to show that for each $I^{\prime} \in \mathcal{I}_{w_{2}}, \mathcal{R}$ contains a box sequence that $(45,15 \theta)$-approximates $\tau_{I^{\prime}}$. So we fix $I^{\prime} \in \mathcal{I}_{w_{2}}$.

The main loop (on $k$ ) of CoveringAlgorithm processes $G$ in overlapping boxes. Since ncost $(\tau) \leq$ $\theta$, one of these boxes, which we'll call $I \times J$, must contain $\tau_{I^{\prime}}$ :

Claim 3.10. Let $I^{\prime} \in \mathcal{I}_{w_{2}}$. There exist intervals $I, J \subseteq \mathbb{N}, I=J$ that are enumerated in the main loop of CoveringAlgorithm such that $I^{\prime} \subseteq I$ and $\tau_{I^{\prime}}$ crosses $G\left(I^{\prime} \times J\right)$.

Proof. Since $\tau$ is of cost at most $\theta$, it cannot use more than $\theta n / 2$ horizontal edges as for each horizontal edge of cost 1 , it must use one vertical edge of cost 1 . Similarly for vertical edges. So $\tau$ is confined to diagonals $\{-\theta n / 2, \ldots, 0, \ldots, \theta n / 2\}$ of $G$. By the choice of $m$ in CoveringAlgorithm, there will be $I$ and $J$ considered in the main loop of the algorithm such that $I^{\prime} \subseteq I$ and $\tau_{I^{\prime}}$ crosses $G(I \times J)$. In particular, $I=J=\{k m, k m+1, \ldots,(k+8) m\}$, where $k \in\left\{0, \ldots, \frac{4}{\theta}-8\right\}$ is the largest integer such that $k m \leq \min \left(I^{\prime}\right)-\frac{\theta n}{2}$ or 0 if $\min \left(I^{\prime}\right)<\frac{\theta n}{2}$, has the desired property.

Let $I, J$ be as provided by the claim. Let $\mathcal{I}^{\prime}$ be the $w_{1}$-decomposition of $I^{\prime}$. We will show one of the following must hold: (1) $\mathcal{R}_{D}$ contains a sequence of certified $w_{1}$-boxes that $(45,15 \theta)$ approximates $\tau_{I^{\prime}}$, or (2) There is a single certified $w_{2}$-box in $\mathcal{R}_{E}$ that $(45,15 \theta)$-approximates $\tau_{I^{\prime}}$.

Let $t=\log \frac{1}{\theta}$. For $i=t, \ldots, 0$, let $\epsilon_{i}=2^{-i}$ and let $\mathcal{S}_{i}$ be the set $\mathcal{S}$ obtained at the iteration $i$ of CoveringAlgorithm $\left(x, y, n, w_{1}, w_{2}, d, \theta\right)$.

We note:

Claim 3.11. Let $i \in\{0, \ldots, \log 1 / \theta\}$. Suppose $I^{\prime \prime} \in \mathcal{I}_{w_{1}}(I)$ and $J^{\prime \prime} \subseteq J$ is $\epsilon_{i} / 8$-aligned. If $I^{\prime \prime} \notin \mathcal{S}_{i}$ and $n \operatorname{cost}\left(I^{\prime \prime} \times J^{\prime \prime}\right) \leq \epsilon_{i}$ then $\left(I^{\prime \prime} \times J^{\prime \prime}, 5 \epsilon_{i}\right) \in \mathcal{R}_{D}$.

Proof. If $I^{\prime \prime} \notin \mathcal{S}_{i}$ then in the call to DenseStripRemoval $\left(G(I \times J), n, w_{1}, d / \epsilon_{i}, \epsilon_{i} / 8, \epsilon_{i}\right)$ there is an iteration of the main loop, where the selected interval $\tilde{I}$ from $\mathcal{T}$ is declared dense and $\Delta_{\text {edit }}\left(x_{\tilde{I}}, x_{I^{\prime \prime}}\right) \leq$ $2 \epsilon_{i}$. Since $\Delta_{\text {edit }}\left(x_{I^{\prime \prime}}, y_{J^{\prime \prime}}\right) \leq \epsilon_{i}, \Delta_{\text {edit }}\left(x_{\tilde{I}}, y_{J^{\prime \prime}}\right) \leq 3 \epsilon_{i}$ and so $I^{\prime \prime} \in \mathcal{X}$ and $J^{\prime \prime} \in \mathcal{Y}$. Thus, DenseStripRemoval certifies $\left(I^{\prime \prime} \times J^{\prime \prime}, 5 \epsilon_{i}\right)$, which is added to $\mathcal{R}_{D}$.

The theorem follows from: 
Claim 3.12. For the interval $I^{\prime} \in \mathcal{I}_{w_{2}}$, assuming successful sampling for $\tau, \mathcal{R}_{E}$ or $\mathcal{R}_{D}$ contains a (45, 150)-approximation of $\tau_{I^{\prime}}$.

The proof is similar to that of Claim 2.1, with adjustments for some technicalities.

Proof. Let $\tau^{\prime}=\tau_{I^{\prime}}$ and $\kappa=\operatorname{ncost}\left(\tau^{\prime}\right)$. Let $\mathcal{I}^{\prime}=\mathcal{I}_{w_{1}}\left(I^{\prime}\right)$. For $I^{\prime \prime} \in \mathcal{I}^{\prime}$, let $\kappa_{I^{\prime \prime}}=\operatorname{ncost}\left(\tau_{I^{\prime \prime}}\right)$. By Proposition 3.4, for all $I^{\prime \prime} \in \mathcal{I}^{\prime}$ and $\epsilon_{i} \geq \kappa_{I^{\prime \prime}}$ there is an $\epsilon_{i} / 8$-aligned vertical interval $J_{i}^{\tau}\left(I^{\prime \prime}\right) \subseteq J$, such that $\operatorname{ncost}\left(I^{\prime \prime} \times J_{i}^{\tau}\left(I^{\prime \prime}\right)\right) \leq 2 \kappa_{I^{\prime \prime}}+\epsilon_{i} / 8$ and $I^{\prime \prime} \times J_{i}^{\tau}\left(I^{\prime \prime}\right)\left(1-\kappa_{I^{\prime \prime}}-\epsilon_{i} / 8\right)$-covers $\tau_{I^{\prime}}$.

Let $s\left(I^{\prime \prime}\right)$ be the largest integer such that $\epsilon_{s\left(I^{\prime \prime}\right)} \geq 3 \kappa_{I^{\prime \prime}}+\kappa+\theta$. Let $t\left(I^{\prime \prime}\right) \leq s\left(I^{\prime \prime}\right)$ be the largest integer such that $I^{\prime \prime} \notin \mathcal{S}_{t\left(I^{\prime \prime}\right)}$. (Since $\theta n / w_{1} \geq d, \mathcal{S}_{0}=\emptyset$, so $t\left(I^{\prime \prime}\right)$ is well-defined.) Let $a\left(I^{\prime \prime}\right)=\epsilon_{s\left(I^{\prime \prime}\right)}$ (this plays a similar role to $u\left(I^{\prime \prime}\right)$ in Section 20) and $b\left(I^{\prime \prime}\right)=\epsilon_{t\left(I^{\prime \prime}\right)}$.

For all $\epsilon_{i} \in\left[a\left(I^{\prime \prime}\right), b\left(I^{\prime \prime}\right)\right], \operatorname{ncost}\left(I^{\prime \prime} \times J_{i}^{\tau}\left(I^{\prime \prime}\right)\right) \leq \epsilon_{i}$ and $I^{\prime \prime} \times J_{i}^{\tau}\left(I^{\prime \prime}\right)\left(1-\epsilon_{i}\right)$-covers $\tau^{\prime}$. By the definition of $b\left(I^{\prime \prime}\right)$ and Claim 3.11, $\mathcal{R}_{D}$ contains the certified box $\left(I^{\prime \prime} \times J_{t\left(I^{\prime \prime}\right)}^{\tau}\left(I^{\prime \prime}\right), 5 b_{I^{\prime \prime}}\right)$. So $\mathcal{R}_{D}$ contains a $(45,15 \theta)$-approximation of $\tau^{\prime}$ if it happens that:

$$
\sum_{I^{\prime \prime} \in \mathcal{I}^{\prime}} 5 b\left(I^{\prime \prime}\right) \leq \frac{45}{8} \sum_{I^{\prime \prime} \in \mathcal{I}^{\prime}} a\left(I^{\prime \prime}\right) .
$$

Indeed, since $a\left(I^{\prime \prime}\right)=\epsilon_{s\left(I^{\prime \prime}\right)} \leq 2\left(3 \kappa_{I^{\prime \prime}}+\kappa+\theta\right), \sum_{I^{\prime \prime} \in \mathcal{I}^{\prime}} \mu\left(I^{\prime \prime}\right)=\mu\left(I^{\prime}\right), \sum_{I^{\prime \prime} \in \mathcal{I}^{\prime}} \kappa_{I^{\prime \prime}} \mu\left(I^{\prime \prime}\right) \leq \kappa \mu\left(I^{\prime}\right)$ (by Proposition 3.2), under Equation 4 being true:

$$
\sum_{I^{\prime \prime} \in \mathcal{I}^{\prime}} 5 b\left(I^{\prime \prime}\right) \mu\left(I^{\prime \prime}\right) \leq \frac{45}{8} \sum_{I^{\prime \prime} \in \mathcal{I}^{\prime}}\left(6 \kappa_{I^{\prime \prime}}+2 \kappa+2 \theta\right) \mu\left(I^{\prime \prime}\right) \leq(45 \kappa+15 \theta) \mu\left(I^{\prime}\right) .
$$

Next we determine a sufficient condition that $\mathcal{R}_{E}$ contains a box sequence (consisting of a single box) that $(5,4 \theta)$-approximates $\tau^{\prime}$. We will show that if this condition does not hold then Equation 4 is true. Let $\mathcal{S}_{i}\left(I^{\prime}\right)=\mathcal{S}_{i} \cap \mathcal{I}^{\prime}$. Interval $I^{\prime \prime} \in \mathcal{S}_{i}\left(I^{\prime}\right)$ is a winner for iteration $i$ if $\epsilon_{i} \geq a\left(I^{\prime \prime}\right)$. This set of winners is denoted by $\mathcal{W}_{i}\left(I^{\prime}\right)$. It suffices that during iteration $i$, the set of $c_{1} \log ^{2} n$ samples taken in SparseStripExtensionSampling includes a winner $I^{\prime \prime}$; then since $\Delta_{\text {edit }}\left(I^{\prime \prime}, J_{i}^{\tau}\left(I^{\prime \prime}\right)\right) \leq \epsilon_{i}$, the (adjusted) diagonal extension $I^{\prime} \times \tilde{J}$ of $I^{\prime \prime} \times J_{i}^{\tau}\left(I^{\prime \prime}\right)$ will be certified. By Proposition 3.5, $I^{\prime} \times \tilde{J}$ has normalized cost at most $3 \kappa+2 \epsilon_{i} w_{1} / w_{2} \leq 3 \kappa+2 \theta \leq 3 \epsilon_{i}$ and it $(1-(\kappa+\theta))$-covers $\tau^{\prime}$. If $\kappa=0$ then $\left(I^{\prime} \times \tilde{J}, \operatorname{ncost}\left(I^{\prime} \times \tilde{J}\right)+\theta+2^{-\log n}\right)$ is in $\mathcal{R}_{E}$ by the behavior of SparseStripExtensionSampling and it $(5,4 \theta)$-approximates $\tau^{\prime}$. Otherwise $\kappa \geq 1 / n$; so set $k=\lfloor\log 1 / \kappa\rfloor$. Thus, $k \leq \log n$ and $2^{-k} \in[\kappa, 2 \kappa)$. Then $\left(I^{\prime} \times \tilde{J}, \operatorname{ncost}\left(I^{\prime} \times \tilde{J}\right)+\theta+2^{-k}\right)$ is in $\mathcal{R}_{E}$ and it $(5,4 \theta)$-approximates $\tau^{\prime}$.

Under successful sampling of extensions for $\tau$ if $\left|\mathcal{W}_{i}\left(I^{\prime}\right)\right| \geq \frac{1}{64}\left|\mathcal{S}_{i}\left(I^{\prime}\right)\right|$, at least one interval from $\mathcal{W}_{i}\left(I^{\prime}\right)$ will be included in our $c_{1} \log ^{2} n$ samples during SparseStripExtensionSampling and $\mathcal{R}_{E}$ will contain a $(5,4 \theta)$-approximation of $\tau^{\prime}$ as above. So suppose this condition is not met, i.e., for all $i$, $\left|\mathcal{W}_{i}\left(I^{\prime}\right)\right|<\frac{1}{64}\left|\mathcal{S}_{i}\left(I^{\prime}\right)\right|$. This implies:

$$
\text { For all } i,\left|\mathcal{W}_{i}\left(I^{\prime}\right)\right|<\frac{1}{32}\left|\mathcal{S}_{i}\left(I^{\prime}\right)-\mathcal{W}_{i}\left(I^{\prime}\right)\right| \text {. }
$$

We show that this in turn implies (44). Multiplying (5) by $\epsilon_{i}$ and summing on $i$ yields:

$$
\sum_{I^{\prime \prime} \in \mathcal{I}^{\prime}} \sum_{i: I^{\prime \prime} \in \mathcal{W}_{i}\left(I^{\prime}\right)} \epsilon_{i}<\frac{1}{32} \sum_{I^{\prime \prime} \in \mathcal{I}^{\prime}} \sum_{i: I^{\prime \prime} \in \mathcal{S}_{i}\left(I^{\prime}\right)-\mathcal{W}_{i}\left(I^{\prime}\right)} \epsilon_{i} .
$$

$I^{\prime \prime} \in \mathcal{S}_{i}\left(I^{\prime}\right)-\mathcal{W}_{i}\left(I^{\prime}\right)$ implies $\epsilon_{i}<a\left(I^{\prime \prime}\right)$. Summing the geometric series:

$$
\sum_{i: I^{\prime \prime} \in \mathcal{S}_{i}\left(I^{\prime}\right)-\mathcal{W}_{i}\left(I^{\prime}\right)} \epsilon_{i} \leq 2 a\left(I^{\prime \prime}\right)
$$


Either $a\left(I^{\prime \prime}\right)=b\left(I^{\prime \prime}\right)$ or $a\left(I^{\prime \prime}\right)<b\left(I^{\prime \prime}\right)$. If the latter, then $I^{\prime \prime} \in \mathcal{W}_{i}\left(I^{\prime}\right)$ for $\epsilon_{i}=b\left(I^{\prime \prime}\right) / 2$. So:

$$
\begin{aligned}
\sum_{I^{\prime \prime} \in \mathcal{I}^{\prime}} b\left(I^{\prime \prime}\right) & \leq \sum_{I^{\prime \prime}}\left(a\left(I^{\prime \prime}\right)+\sum_{i: I^{\prime \prime} \in \mathcal{W}_{i}\left(I^{\prime}\right)} 2 \epsilon_{i}\right) \\
& <\sum_{I^{\prime \prime}}\left(a\left(I^{\prime \prime}\right)+\frac{1}{16} \sum_{i: I^{\prime \prime} \in \mathcal{S}_{i}\left(I^{\prime}\right)-\mathcal{W}_{i}\left(I^{\prime}\right)} \epsilon_{i}\right) \\
& \leq \frac{9}{8} \sum_{I^{\prime \prime} \in \mathcal{I}^{\prime}} a\left(I^{\prime \prime}\right)
\end{aligned}
$$

which implies Equation 4. (The second inequality follows from (6) and the last inequality from (77).)

\subsection{Time complexity of CoveringAlgorithm}

We write $t(w, \epsilon)$ for the time of $\operatorname{SMALL-ED}\left(z_{1}, z_{2}, \epsilon\right)$ on strings of length $w$. We assume $t(w, \epsilon) \geq$ $w$, and that for $k \geq 1$, there is a constant $c(k)$ such that for all $\epsilon \in[0,1]$ and all $w>1, t(w, k \epsilon) \leq$ $c(k) \cdot t(w, \epsilon)+c(k)$. As mentioned earlier, by [26], we can use $t(w, \epsilon)=O\left(w^{2} \epsilon\right)$.

Theorem 3.13. Let $n$ be a sufficiently large power of 2 and $\theta \in[1 / n, 1]$ be a power of 2. Let $x, y$ be strings of length $n$. Let $\log n \leq w_{1} \leq w_{2} \leq \theta n / 4,1 \leq d \leq n$ be powers of 2 , where $w_{1} \mid w_{2}$ and $w_{2} \mid n$, and $w_{1} / w_{2} \leq \theta$. The size of the set $\mathcal{R}$ output by CoveringAlgorithm is $O\left(\left(\frac{n}{w_{1}}\right)^{2} \log ^{2} n\right)$ and in any run that satisfies successful classification sampling, CoveringAlgorithm runs in time:

$$
O\left(|\mathcal{R}|+\sum_{\substack{i=\log 1 / \theta, \ldots, 0 \\ \epsilon=2^{-i}}}\left(\frac{\theta n^{2} \log n}{d \epsilon w_{1}^{2}} \cdot t\left(w_{1}, \epsilon\right)+\frac{\theta n^{2} \log ^{2} n}{w_{1} w_{2} \epsilon} \cdot t\left(w_{1}, \epsilon\right)+\frac{n d \log ^{2} n}{w_{2} \epsilon} \cdot t\left(w_{2}, \epsilon\right)\right)\right)
$$

Proof. To bound $|\mathcal{R}|$ note that for each choice of $k, i$ in the outer and inner loops of CoveringAlgorithm, the set of candidate boxes of width $w_{1}$ has size $O\left(\frac{\theta n}{w_{1}} \frac{\theta n}{w_{1} \epsilon_{i}}\right)$. This upper bounds the number of boxes certified by DenseStripRemoval. The call to SparseStripExtensionSampling constructs at most one diagonal extension for each such candidate box, and each diagonal extension gives rise to at most $O(\log n)$ certified boxes. Thus, for each $(k, i)$ there are $O\left(\frac{\theta^{2} n^{2} \log n}{\left(w_{1}\right)^{2} \epsilon_{i}}\right)$ certified boxes. Summing the geometric series over $i$, noting that $\min \left(\epsilon_{i}\right)=\theta$, and summing over $O(1 / \theta)$ values of $k$ gives the required bound on $|\mathcal{R}|$.

The steps in the algorithm that actually construct certified boxes cost $O(1)$ per box giving the first term in the time bound.

We next bound the other contributions to the running time. The outer loop of CoveringAlgorithm has $\frac{4}{\theta}-7$ iterations on $k$ 's. The inner loop has $1+\log \frac{1}{\theta}$ iterations on $i$. Each iteration invokes DenseStripRemoval and SparseStripExtensionSampling on $I \times J$ with $I$ and $J$ of width at most $4 \theta n$.

We bound the time of a call to DenseStripRemoval. To distinguish between local variables of DenseStripRemoval and global variables of CoveringAlgorithm, we denote local input variables as $\hat{G}, \hat{n}, \hat{w}, \hat{d}, \hat{\delta}, \hat{\epsilon}$. For $\mathcal{B}$ and $\mathcal{T}$ as in DenseStripRemoval, $|\mathcal{T}| \leq|\mathcal{B}| \leq \frac{\mu\left(I_{\hat{G}}\right)}{\hat{\delta} \hat{w}}$, since $\mu\left(I_{\hat{G}}\right)=\mu\left(J_{\hat{G}}\right)$. The main while loop of DenseStripRemoval repeatedly picks intervals $I \in \mathcal{T}$ and samples $c_{0}|\mathcal{B}| \frac{\log \hat{n}}{\hat{d}} \leq$ 
$\frac{c_{0} \mu\left(I_{\hat{G}}\right) \log \hat{n}}{\hat{d} \hat{\delta} \hat{w}}$ vertical intervals $J$ and tests whether $\Delta_{\text {edit }}\left(x_{I}, y_{J}\right) \leq \hat{\epsilon}$. Each such test takes time $t(\hat{w}, \hat{\epsilon})$. This is done at most once for each of the $\mu\left(I_{\hat{G}}\right) / \hat{w}$ horizontal candidates for a total time of $O\left(\frac{\mu\left(I_{\hat{G}}\right)^{2} \log \hat{n}}{\hat{w}^{2} \hat{\delta} \hat{d}}\right) t(\hat{w}, \hat{\epsilon})$. We next bound the cost of processing a pivot $I$. This requires testing $\Delta_{\text {edit }}\left(x_{I}, y_{J}\right) \leq 3 \hat{\epsilon}$ for $J \in \mathcal{B}$ and $\Delta_{\text {edit }}\left(x_{I}, x_{I^{\prime}}\right) \leq 2 \hat{\epsilon}$ for $I^{\prime} \in \mathcal{T}$. Each test costs $O(t(\hat{w}, \hat{\epsilon})$ ) (by our assumption on $t(\cdot, \cdot))$, and since $|\mathcal{T}| \leq|\mathcal{B}|=\frac{\mu\left(I_{\hat{G}}\right)}{\hat{w} \hat{\delta}}, I$ is processed in time $O\left(\frac{\mu\left(I_{\hat{G}}\right)}{\hat{w} \hat{\delta}} t(\hat{w}, \hat{\epsilon})\right)$. This is multiplied by the number of intervals declared dense, which we now upper bound. If $I$ is declared dense then at the end of processing $I, \mathcal{X}$ is removed from $\mathcal{T}$. This ensures $\Delta_{\text {edit }}\left(I, I^{\prime}\right)>2 \epsilon$ for any two intervals $I, I^{\prime}$ declared dense. By the triangle inequality the sets $\mathcal{B}(I)=\left\{J \in \mathcal{B} ; \Delta_{\text {edit }}\left(x_{I}, y_{J}\right) \leq\right.$ $\epsilon\}$ are disjoint for different pivots. By successful classification sampling, for each pivot $I,|\mathcal{B}(I)| \geq \frac{\hat{d}}{4}$, and thus at most $|\mathcal{B}| /(\hat{d} / 4)=\frac{4 \mu\left(I_{\hat{G}}\right)}{\hat{d} \hat{\delta} \hat{w}}$ intervals are declared dense, so all intervals declared dense are processed in time $O\left(\frac{\mu\left(I_{\hat{G}}\right)^{2}}{\hat{w}^{2} \hat{d} \hat{\delta}^{2}}\right) t(\hat{w}, \hat{\epsilon})$.

The time for dense/sparse classification of intervals and for processing intervals declared dense is at most $O\left(\frac{\mu\left(I_{\hat{G}}\right)^{2} \log \hat{n}}{\hat{w}^{2} \hat{d}^{2}}\right) t(\hat{w}, \hat{\epsilon})$. During iteration $i$ of the inner loop of CoveringAlgorithm, the local variables of DenseStripRemoval are set as $\hat{n}=n, \mu\left(I_{\hat{G}}\right) \leq 4 \theta n, \hat{w}=w_{1}, \hat{d}=d / \epsilon_{i}, \hat{\delta}=\epsilon_{i} / 8$. Substituting these parameters yields time $O\left(\frac{\theta^{2} n^{2} \log n}{\left(w_{1}\right)^{2} d \epsilon_{i}}\right) t\left(w_{1}, \epsilon_{i}\right)$. Multiplying by the $O(1 / \theta)$ iterations on $k$ gives the first summand of the theorem.

Next we turn to SparseStripExtensionSampling. The local input variables $n, w_{1}, w_{2}, \mathcal{S}, \theta$ are set to their global values so we denote them without ^. The other local input variables are denoted as $\hat{G}, \hat{d}, \hat{\delta}, \hat{\epsilon}$. The local variable $\mathcal{B}$ has size $\frac{\mu\left(I_{\hat{G}}\right)}{\hat{\delta} w_{1}}$. By successful classification sampling, we assume that on every call, every interval in $\mathcal{S}$ is $(\hat{d}, \hat{\delta}, \hat{\epsilon})$ - sparse. The outer loop enumerates the $\mu\left(I_{\hat{G}}\right) / w_{2}$ intervals $I^{\prime}$ of $\mathcal{I}_{w_{2}}\left(I_{\hat{G}}\right)$. We select $\mathcal{H}$ to be $c_{1} \log ^{2} n$ random subsets from subsets of $I^{\prime}$ belonging to $\mathcal{S}$. For each $I \in \mathcal{H}$ and $J \in \mathcal{B}$, we call SMALL-ED $\left(x_{I}, y_{J}, \hat{\epsilon}\right)$, taking time $t\left(w_{1}, \hat{\epsilon}\right)$. The total time of all tests is $O\left(\frac{\mu\left(I_{\hat{\hat{C}}}\right)^{2} \log ^{2} n}{\hat{\delta} w_{1} w_{2}}\right) t\left(w_{1}, \hat{\epsilon}\right)$. Using $\hat{d}=d / \epsilon_{i}, \hat{\delta}=\epsilon_{i} / 8$ and $\hat{\epsilon}=\epsilon_{i}$ from the $i$ th call to SparseStripExtensionSampling gives $O\left(\frac{\theta^{2} n^{2} \log ^{2} n}{\epsilon_{i} w_{1} w_{2}}\right) t\left(w_{1}, \epsilon_{i}\right)$. Multiplying by the $O(1 / \theta)$ iterations on $k$ gives the second summand in the theorem.

Assuming successful classification sampling, all intervals in the set $\mathcal{S}$ passed from DenseStripRemoval to SparseStripExtensionSampling are $(\hat{d}, \hat{\delta}, \hat{\epsilon})$-sparse. Therefore, for each sampled $I$, at most $\hat{d}$ intervals $J$ are within $\hat{\epsilon}$ of $I$. For each of these we do a diagonal extension of $I \times J$ to a $w_{2}$-box $I^{\prime} \times J^{\prime}$, and call SMALL-ED $\left(x_{I^{\prime}}, y_{J^{\prime}}, 3 \hat{\epsilon}\right)$ at cost $O\left(t\left(w_{2}, \hat{\epsilon}\right)\right)$ for each call. The number of such calls is $O\left(\frac{\mu\left(I_{\hat{G}}\right) \hat{d} \log ^{2} n}{w_{2}}\right)$. Using the parameter $\hat{d}=d / \epsilon_{i}$ in the $i$ th call of the inner iteration of CoveringAlgorithm, we get a cost of $O\left(\frac{\theta n d \log ^{2} n}{\epsilon_{i} w_{2}}\right) t\left(w_{2}, \epsilon_{i}\right)$ and multiplying by the $O(1 / \theta)$ gives the third summand in the theorem.

Choosing the parameters to minimize the maximum term in the time bound, subject to the restrictions of the theorem and using $t(w, \epsilon)=O\left(\epsilon w^{2}\right)$ we have:

Corollary 3.14. For all sufficient large $n$, and for $\theta \geq n^{-1 / 5}$ (both powers of 2) choosing $w_{1}, w_{2}$, and $d$ to be the largest powers of two satisfying: $w_{1} \leq \theta^{-2 / 7} n^{1 / 7}, w_{2} \leq \theta^{1 / 7} n^{3 / 7}$, and $d \leq \theta^{3 / 7} n^{2 / 7}$, with probability at least $1-n^{-1 / 7}$, CoveringAlgorithm runs in time $\tilde{O}\left(n^{12 / 7} \theta^{4 / 7}\right)$, and outputs the set $\mathcal{R}$ of size at most $\tilde{O}\left(n^{12 / 7} \theta^{4 / 7}\right)$.

Proof. Use the algorithm of [26] that gives $t(w, \epsilon)=O\left(\epsilon w^{2}\right)$. It is routine to check that these choices 
satisfy the requirements of Theorem 3.13 , and also that all three terms in the time analysis, and the number of boxes are all bounded by the claimed bound.

\section{Min-cost Paths in Shortcut Graphs}

We now describe the second phase of our algorithm, which uses the set $\mathcal{R}$ output by CoveringAlgorithm to upper bound $d_{\text {edit }}(x, y)$. A shortcut graph on vertex set $\{0, \ldots, n\} \times\{0, \ldots, n\}$ consists of the $\mathrm{H}$ and $\mathrm{V}$ edges of cost 1 , together with an arbitrary collection of shortcut edges $(i, j) \rightarrow\left(i^{\prime}, j^{\prime}\right)$ where $i<i^{\prime}$ and $j<j^{\prime}$, also denoted by $e_{I, J}$ where $I=\left\{i, \ldots, i^{\prime}\right\}$ and $J=\left\{j, \ldots, j^{\prime}\right\}$, along with their costs. A certified graph (for $x, y$ ) is a shortcut graph where every shortcut edge $e_{I, J}$ has cost at least $d_{\text {edit }}\left(x_{I}, y_{J}\right)$. The min-cost path from $(0,0)$ to $(n, n)$ in a certified graph upper bounds $d_{\text {edit }}(x, y)$. The second phase algorithm uses $\mathcal{R}$ to construct a certified graph, and computes the min-cost path to upper bound on $d_{\text {edit }}(x, y)$.

A certified box $(I \times J, \kappa)$ corresponds to the $e_{I, J}$ with cost $\kappa \mu(I)$. (In the certified graph we use non-normalized costs.) However, the certified graph built from $\mathcal{R}$ in this way may not have a path of cost $O\left(d_{\text {edit }}(x, y)+\theta n\right)$. We need a modified conversion of $(I \times J, \kappa)$. If $\kappa \geq 1 / 2$ we add no shortcut. Otherwise $(I \times J, \kappa)$ converts to the edge $e_{I, J^{\prime}}$ with cost $3 \kappa \mu(I)$ where $J^{\prime}$ is obtained by shrinking $J: \min \left(J^{\prime}\right)=\min (J)+\ell$ and $\max \left(J^{\prime}\right)=\max \left(J^{\prime}\right)-\ell$ where $\ell=\lfloor\kappa \mu(I)\rfloor$. By Proposition 3.1, this is a certified edge. Call the resulting graph $\widetilde{G}$. We claim:

Lemma 4.1. Let $\tau$ be a source-sink path in $G_{x, y}$. If $\mathcal{R}$ contains a sequence $\sigma$ that $(k, \theta)$-approximates $\tau$ then there is a source-sink path $\tau^{\prime}$ in $\widetilde{G}$ that consists of the shortcuts corresponding to $\sigma$ together with some $H$ and $V$ edges with $\operatorname{cost}_{\widetilde{G}}\left(\tau^{\prime}\right) \leq 5\left(k \cdot \operatorname{cost}_{G_{x, y}}(\tau)+\theta n\right)$.

Proof. We will modify the path $\tau$ in $G_{x, y}$ to a path $\tau^{\prime}$ in $\widetilde{G}$ of comparable cost. Let $\left\{\left(I_{1} \times J_{1}, \epsilon_{1}\right),\left(I_{2} \times\right.\right.$ $\left.\left.J_{2}, \epsilon_{2}\right), \ldots,\left(I_{m} \times J_{m}, \epsilon_{m}\right)\right\}$ be the set of certified boxes that $(k, \theta)$-approximates $\tau$. Let $\ell_{i}=\left\lfloor\mu\left(I_{i}\right) \cdot \epsilon_{i}\right\rfloor$. Let $L$ be the subset of $[m]$ for which $\epsilon_{i} \leq 1 / 2$. For $i \in L$, let $e_{i}=e_{I_{i}, J_{i}^{\prime}}$ be the shortcut edge with weight $3 \epsilon_{i}$. We claim (1) there is a source-sink path in $\widetilde{G}$ that consists of $\left\{e_{i}: i \in L\right\}$ together with a horizontal path $H_{i}$ whose projection to the $x$-axis is $I_{i}$ for each $i \in[m]-L$, and a collection of (possibly empty) vertical paths $V_{0}, V_{1}, \ldots, V_{m}$ where the $x$-coordinate of $V_{i}$ for $i>0$ is $\max \left(I_{i}\right)$ and 0 for $V_{0}$, and (2) its cost satisfies the bound of the lemma.

For the first claim, define for $h \in[m], p_{h}=\left(i_{h}, j_{h}\right)$ to be the first point in $\tau_{I_{h}}$ and define $p_{m+1}=$ $(n, n)$. We will define $\tau^{\prime}$ to pass through all of the $p_{h}$. In preparation, observe that for $h \in L$, since $I_{h} \times J_{h}\left(1-\epsilon_{h}\right)$-covers $\tau$, we have $\min \left(J_{h}^{\prime}\right)=\min \left(J_{h}\right)+\ell_{h} \geq j_{h}$ and $\max \left(J_{h}^{\prime}\right)=\max (J)-\ell_{h} \leq j_{h+1}$. Define the portion $\tau_{h}^{\prime}$ between $p_{h}$ and $p_{h+1}$ by climbing vertically from $p_{h}$ to $\left(i_{h}, \min \left(J_{h}^{\prime}\right)\right)$ and if $h \in L$ traversing $e_{I_{h}, J_{h}^{\prime}}$ and climbing to $p_{h+1}$ and if $h \notin L$ then move horizontally from $\left(i_{h}, \min \left(J_{h}^{\prime}\right)\right)$ to $\left(i_{h+1}, \min \left(J_{h}^{\prime}\right)\right)$ and then climb to $p_{h+1}$.

For the second claim, we upper bound $\operatorname{cost}\left(\tau^{\prime}\right)$. For $h \in L, e_{I_{h}, J_{h}} \operatorname{costs} 3 \ell_{h}$, and for $h \notin L$, the horizontal path that projects to $I_{h} \operatorname{costs} \mu\left(I_{h}\right) \leq 2 \ell_{h}$; the total is at most $\sum_{h} 3 \ell_{h}$. The cost of vertical edges is $n-\sum_{h \in L} \mu\left(J_{h}^{\prime}\right)=\sum_{h \in L}\left(\mu\left(J_{h}\right)-\mu\left(J_{h}^{\prime}\right)\right)+\sum_{h \notin L} \mu\left(J_{h}\right)=\sum_{h \in L} 2 \ell_{h}+\sum_{h \notin L} \mu\left(J_{h}\right) \leq \sum_{h} 2 \ell_{h}$, since $\sum_{h} \mu\left(J_{h}\right)=\sum_{h} \mu\left(I_{h}\right)=n$. So $\operatorname{cost}\left(\tau^{\prime}\right) \leq \sum_{h} 5 \ell_{h}$. Since $\sum_{i=1}^{m} \ell_{i} \leq k \cdot \operatorname{cost}_{G_{x, y}}(\tau)+\theta \cdot n$ by definition of $(k, \theta)$-approximation, the lemma follows.

Computing the min-cost. We present an $O(n+m \log (m n))$ algorithm to find a min-cost sourcesink path in a shortcut graph $\widetilde{G}$ with $m$ shortcuts. It's easier to switch to the max-benefit problem: Let $\widetilde{H}$ be the same graph with $\operatorname{cost} c_{e}$ of $e=(i, j) \rightarrow\left(i^{\prime}, j^{\prime}\right)$ replaced by benefit $b_{e}=\left(i^{\prime}-i\right)+\left(j^{\prime}-\right.$ 
$j)-c_{e}$, (so $\mathrm{H}$ and $\mathrm{V}$ edges have benefit 0 ). The min-cost path of $\widetilde{G}$ is $2 n$ minus the max-benefit path of $\widetilde{H}$. To compute the max-benefit path of $\widetilde{H}$, we use a binary tree data structure with leaves $\{1, \ldots, n\}$, where each node $v$ stores a number $b_{v}$, and a collection of lists $L_{1}, \ldots, L_{n}$, where $L_{i}$ stores pairs $(e, q(e))$ where the head of $e$ has $x$-coordinate $i$ and $q(e)$ is the max benefit of a path that ends with $e$.

We proceed in $n-1$ rounds. Let the set $A_{i}$ consist of all the shortcuts whose tail has $x$-coordinate $i$. The preconditions for round $i$ are: (1) for each leaf $j$, the stored value $b_{j}$ is the max benefit path to $(i, j)$ that includes a shortcut whose head has $y$-coordinate $j$ (or 0 if there is no such path), (2) for each internal node $v, b_{v}=\max \left\{b_{j}: j\right.$ is a leaf in the subtree of $\left.v\right\}$, and (3) for every edge $e=\left(i^{\prime}, j^{\prime}\right) \rightarrow\left(i^{\prime \prime}, j^{\prime \prime}\right)$ with $i^{\prime}<i$, the value $q(e)$ has been computed and $(e, q(e))$ is in list $L_{i^{\prime \prime}}$. During round $i$, for each shortcut $e=(i, j) \rightarrow\left(i^{\prime}, j^{\prime}\right)$ in $A_{i}, q(e)$ equals the max of $b_{v}+b_{e}$ over tree leaves $v$ with $v \leq j$. This can be computed in $O(\log n)$ time as $\max b_{v}+b_{e}$, over $\{j\}$ union the set of left children of vertices on the root-to- $j$ path that are not themselves on the path. Add $(e, q(e))$ to list $L_{i^{\prime}}$. After processing $A_{i}$, update the binary tree: for each $(e, q(e)) \in L_{i+1}$, let $j$ be the $y$-coordinate of the head of $e$ and for all vertices $v$ on the root-to- $j$ path, replace $b_{v}$ by $\max \left(b_{v}, q(e)\right)$. The tree then satisfies the precondition for round $i+1$. The output of the algorithm is $b_{n}$ at the end of round $n-1$. It takes $O(n)$ time to set up the data structure, $O(m \log m)$ time to sort the shortcuts, and $O(\log n)$ processing time per shortcut (computing $q(e)$ and later updating the data structure).

\section{$5 \quad$ Summing up and speeding up}

To summarize, the algorithm GAP-UB ${ }_{\theta}$ runs CoveringAlgorithm of Section 3, converts the output into a shortcut graph, and runs the min-cost path algorithm of Section 4. By Corollary 3.14, and the quasilinear running time (in the number of shortcuts) of the min-cost path algorithm, the algorithm GAP-UB $\mathbf{U}_{\theta}$ runs in time $\tilde{O}\left(n^{12 / 7} \theta^{4 / 7}\right)$. The construction of the main algorithm ED-UB from GAP-UB is standard:

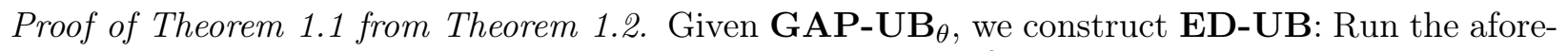
mentioned exact algorithm of [21] with running time $O\left(n+k^{2}\right)$ time on instances of edit distance $k$, for $O\left(n+n^{2-2 / 5}\right)$ time. If it terminates then it outputs the exact edit distance. Otherwise, the failure to terminate implies $d_{\text {edit }}(x, y) \geq n^{4 / 5}$. Now run $\mathbf{G A P}-\mathbf{U B}_{\theta_{j}}(x, y)$ for $\theta_{j}=(1 / 2)^{j}$ for $j=\left\{0, \ldots, \frac{\log n}{5}\right\}$ and output the minimum of all upper bounds obtained. Let $j$ be the largest index with $\theta_{j} n \geq d_{\text {edit }}(x, y)$ (such an index exists since $j=0$ works). The output is at most $840 \theta_{j} n \leq 1680 d_{\text {edit }}(x, y)$. We run at most $O(\log n)$ iterations, each with running time $\tilde{O}\left(n^{2-2 / 7}\right)$.

Speeding up the algorithm. The running time of ED-UB is dominated by the cost of SMALL-ED( $\left.z_{1}, z_{2}, \epsilon\right)$ on pairs of strings of length $w \in\left\{w_{1}, w_{2}\right\}$. We use Ukkonen's algorithm [26] with $t(w, \epsilon)=O\left(\epsilon w^{2}\right)$. By replacing Ukkonen's algorithm with ED-UB we can get a revised algorithm ED-UB $\mathbf{U}_{1}$. This worsens the approximation factor (roughly multiplying it by the approximation factor of ED-UB) but improves running time. The internal parameters $w_{1}, w_{2}, d$ have to be adjusted to maximize savings. One can iterate this process any constant number of times to get faster algorithms with worse (but still constant) approximation factors. Because of the dependence of the analysis on $\theta$, one does not get a faster edit distance algorithm for all $\theta \in[0,1]$ but only for $\theta$ close to 1 . This approach combined with further ideas is used in [15,20]. 
Acknowledgements We thank the FOCS 2018 committee and especially several anonymous referees for helpful comments and suggestions. Michael Saks thanks C. Seshadhri for insightful discussions on the edit distance problem. The research leading to these results is partially supported by the Grant Agency of the Czech Republic under the grant agreement no. 19-27871X, by the H2020-MSCA-RISE project CoSP-GA no. 823748, and by the European Research Council under the European Union's Seventh Framework Programme (FP/2007-2013)/ERC Grant Agreement no. 616787. The research is also supported in part by Simons Foundation under grant 332622 .

\section{References}

[1] Amir Abboud and Arturs Backurs. Towards hardness of approximation for polynomial time problems. In 8th Innovations in Theoretical Computer Science Conference, ITCS 2017, January 9-11, 2017, Berkeley, CA, USA, pages 11:1-11:26, 2017.

[2] Amir Abboud, Arturs Backurs, and Virginia Vassilevska Williams. Tight hardness results for LCS and other sequence similarity measures. In IEEE 56th Annual Symposium on Foundations of Computer Science, FOCS 2015, Berkeley, CA, USA, 17-20 October, 2015, pages 59-78, 2015.

[3] Amir Abboud, Thomas Dueholm Hansen, Virginia Vassilevska Williams, and Ryan Williams. Simulating branching programs with edit distance and friends: or: a polylog shaved is a lower bound made. In Proceedings of the 48th Annual ACM SIGACT Symposium on Theory of Computing, STOC 2016, Cambridge, MA, USA, June 18-21, 2016, pages 375-388, 2016.

[4] Alexandr Andoni. Simpler constant-factor approximation to edit distance problems. Manuscript, 2018.

[5] Alexandr Andoni, Robert Krauthgamer, and Krzysztof Onak. Polylogarithmic approximation for edit distance and the asymmetric query complexity. In 51th Annual IEEE Symposium on Foundations of Computer Science, FOCS 2010, October 23-26, 2010, Las Vegas, Nevada, USA, pages 377-386, 2010.

[6] Alexandr Andoni and Huy L. Nguyen. Near-optimal sublinear time algorithms for Ulam distance. In Proceedings of the Twenty-First Annual ACM-SIAM Symposium on Discrete Algorithms, SODA 2010, Austin, Texas, USA, January 17-19, 2010, pages 76-86, 2010. doi:10.1137/1.9781611973075.8.

[7] Alexandr Andoni and Negev Shekel Nosatzki. Edit distance in near-linear time: it's a constant factor. In 61th Annual IEEE Symposium on Foundations of Computer Science, FOCS 2020, page to appear, 2020.

[8] Alexandr Andoni and Krzysztof Onak. Approximating edit distance in near-linear time. In Proceedings of the Forty-first Annual ACM Symposium on Theory of Computing, STOC '09, pages 199-204, New York, NY, USA, 2009. ACM.

[9] Arturs Backurs and Piotr Indyk. Edit distance cannot be computed in strongly subquadratic time (unless SETH is false). In Proceedings of the Forty-Seventh Annual ACM on Symposium on Theory of Computing, STOC '15, pages 51-58, New York, NY, USA, 2015. ACM. 
[10] Z. Bar-Yossef, T.S. Jayram, R. Krauthgamer, and R. Kumar. Approximating edit distance efficiently. In Foundations of Computer Science, 2004. Proceedings. 45th Annual IEEE Symposium on, pages 550-559, Oct 2004.

[11] Tugkan Batu, Funda Ergün, Joe Kilian, Avner Magen, Sofya Raskhodnikova, Ronitt Rubinfeld, and Rahul Sami. A sublinear algorithm for weakly approximating edit distance. In Proceedings of the Thirty-fifth Annual ACM Symposium on Theory of Computing, STOC '03, pages 316324, New York, NY, USA, 2003. ACM.

[12] Tuğkan Batu, Funda Ergun, and Cenk Sahinalp. Oblivious string embeddings and edit distance approximations. In Proceedings of the Seventeenth Annual ACM-SIAM Symposium on Discrete Algorithm, SODA '06, pages 792-801, Philadelphia, PA, USA, 2006. Society for Industrial and Applied Mathematics.

[13] Mahdi Boroujeni, Soheil Ehsani, Mohammad Ghodsi, Mohammad Taghi Hajiaghayi, and Saeed Seddighin. Approximating edit distance in truly subquadratic time: Quantum and MapReduce. In Proceedings of the Twenty-Ninth Annual ACM-SIAM Symposium on Discrete Algorithms, SODA 2018, New Orleans, LA, USA, January 7-10, 2018, pages 1170-1189, 2018.

[14] Mahdi Boroujeni, Soheil Ehsani, Mohammad Ghodsi, Mohammad Taghi Hajiaghayi, and Saeed Seddighin. Approximating edit distance in truly subquadratic time: Quantum and MapReduce (extended version of [13]). 2018.

[15] Joshua Brakensiek and Aviad Rubinstein. Constant-factor approximation of near-linear edit distance in near-linear time. In Proccedings of the 52nd Annual ACM SIGACT Symposium on Theory of Computing, STOC 2020, pages 685-698. ACM, 2020.

[16] Karl Bringmann and Marvin Künnemann. Quadratic conditional lower bounds for string problems and dynamic time warping. In IEEE 56th Annual Symposium on Foundations of Computer Science, FOCS 2015, Berkeley, CA, USA, 17-20 October, 2015, pages 79-97, 2015.

[17] Diptarka Chakraborty, Elazar Goldenberg, and Michal Koucký. Streaming algorithms for computing edit distance without exploiting suffix trees. CoRR, abs/1607.03718, 2016. arXiv:1607.03718

[18] Diptarka Chakraborty, Elazar Goldenberg, and Michal Koucký. Streaming algorithms for embedding and computing edit distance in the low distance regime. In Proceedings of the 48th Annual ACM SIGACT Symposium on Theory of Computing, STOC 2016, Cambridge, MA, USA, June 18-21, 2016, pages 712-725, 2016.

[19] Szymon Grabowski. New tabulation and sparse dynamic programming based techniques for sequence similarity problems. Discrete Applied Mathematics, 212:96-103, 2016.

[20] Michal Koucký and Michael E. Saks. Constant factor approximations to edit distance on far input pairs in nearly linear time. In Proccedings of the 52nd Annual ACM SIGACT Symposium on Theory of Computing, STOC 2020, pages 699-712. ACM, 2020.

[21] Gad M. Landau, Eugene W. Myers, and Jeanette P. Schmidt. Incremental string comparison. SIAM J. Comput., 27(2):557-582, April 1998. 
[22] VI Levenshtein. Binary Codes Capable of Correcting Deletions, Insertions and Reversals. Soviet Physics Doklady, 10:707, 1966.

[23] William J. Masek and Michael S. Paterson. A faster algorithm computing string edit distances. Journal of Computer and System Sciences, 20(1):18 - 31, 1980.

[24] Timothy Naumovitz, Michael E. Saks, and C. Seshadhri. Accurate and nearly optimal sublinear approximations to Ulam distance. In Proceedings of the Twenty-Eighth Annual ACM-SIAM Symposium on Discrete Algorithms, SODA 2017, Barcelona, Spain, Hotel Porta Fira, January 16-19, pages 2012-2031, 2017.

[25] Barna Saha. The dyck language edit distance problem in near-linear time. In 55th IEEE Annual Symposium on Foundations of Computer Science, FOCS 2014, Philadelphia, PA, USA, October 18-21, 2014, pages 611-620, 2014.

[26] Esko Ukkonen. Algorithms for approximate string matching. Inf. Control, 64(1-3):100-118, March 1985.

[27] Robert A. Wagner and Michael J. Fischer. The string-to-string correction problem. J. ACM, 21(1):168-173, January 1974. 